\title{
Strong convergence in the $p$ th-mean of an averaging principle for two-time-scales SPDEs with jumps
}

\author{
Qing Guo ${ }^{1 * \dagger}$, Peirong Guo ${ }^{2 \dagger}$ and Fangyi Wan ${ }^{1 \dagger}$
}

"Correspondence: gq@nwpu.edu.cn 'School of Aeronautics, Northwestern Polytechnical University, Xi'an, 710072, China Full list of author information is available at the end of the article ${ }^{\dagger}$ Equal contributors

\section{然 Springer}

\begin{abstract}
The main goal of this work is to study an averaging principle for two-time-scales stochastic partial differential equations with jumps. The solutions of reduced equations with modified coefficients are derived to approximate the slow component of the original equation under suitable conditions. It is shown that the slow component can strongly converge to the solution of the corresponding reduced equation in the pth-mean. Our key and novel idea is how to cope with the changes caused by jumps and higher order moments.
\end{abstract}

Keywords: strong convergence in pth-mean; two-time-scales; SPDEs; jumps; averaging principle

\section{Introduction}

In practical science and engineering, many complex systems can be described as singularly perturbed systems with separated two-time-scales driven by random perturbations, for example, chemical reaction dynamics [1], electronic circuits [2] and laser systems [3]. In most cases, people are only interested in investigating the time evolution of the slow component, but that cannot be done directly, unless we solve the full two-time-scales equations. Although computers are now very advanced, they cannot deal with such a disparity of scales. Averaging methods can reduce the computational load. In view of this, the averaging principle, which is an effective tool to analyze the two-time-scales dynamical systems with random perturbations, becomes more and more important and popular to be applied to reduce the dimensions of the original systems.

The theory of the averaging principle has a long and rich history. Let us mention a few of them. Khasminskii [4] first proved the averaging principle of stochastic differential equations (SDEs) driven by Brownian noise. Since then, the averaging principle has been an active research field on which there is a great deal of literature. Freidlin and Wentzell [5] provided a mathematically rigorous overview of fundamental stochastic averaging methods. Golec and Ladde [6] and Xu et al. [7-14] proposed the averaging principle to stochastic dynamical systems in the sense of the mean-square, which implies the convergence in probability. Furthermore, $\mathbb{L}^{2}$-strong convergence (also called mean-square-strong convergence) in averaging principles for several types of slow-fast stochastic dynamical systems

(c) The Author(s) 2017. This article is distributed under the terms of the Creative Commons Attribution 4.0 International License (http://creativecommons.org/licenses/by/4.0/), which permits unrestricted use, distribution, and reproduction in any medium, provided you give appropriate credit to the original author(s) and the source, provide a link to the Creative Commons license, and indicate if changes were made. 
driven by Brownian noise has been investigated by Freidlin [5], Golec [15], Wang [16], and Fu et al. $[17,18]$.

In some circumstances, jump type perturbations can capture some large moves and unpredictable events in such diverse areas as mathematics, finance, statistical physics and life sciences [19-34], while purely Brownian type perturbations cannot do so. It is well known that stochastic partial differential equations (SPDEs) driven by jump type perturbations may be more appropriate to model a great amount of complex systems, which are widely used to describe many interesting phenomena in the fields of physics, biology, chemistry, economics, finance and others [35-41]. Up to now, many scholars have extensively investigated the existence and uniqueness for solutions of SPDEs driven by jump type perturbations. For example, Albeverio et al. [42] investigated the existence and uniqueness of mild solutions to stochastic heat equations driven by Poisson jumps. Hausenblas [43] considered the existence and uniqueness of mild solutions to SPDEs of the jump type. A series of useful theories and methods have been presented to explore SPDEs driven by jumps (see $[19,39])$, and among them, the averaging method has been an important and useful tool to reduce SPDEs driven by jumps. Givon [44] established an averaging principle for twotime-scales jump-diffusion processes in the sense of the mean-square. Quite recently, Xu and Miao [45] established a $\mathbb{L}^{2}$-strong averaging principle for slow-fast SPDEs driven by Poisson random measures. Pei et al. [46] considered the averaging principle for stochastic hyperbolic- parabolic equations driven by Poisson random measures with slow and fast time-scales.

However, the work on the averaging principle mainly discussed $\mathbb{L}^{2}$-strong convergence for two-time-scales jump-diffusion processes, which does not involve $\mathbb{L}^{p}(p>2)$-strong convergence in general. Generally, people need to estimate the higher order moments which possess a good robustness and can be applied in computations in statistics, finance and other fields. To the best of the authors' knowledge, the averaging principle for twotime-scales SPDEs with jumps has not been considered in $\mathbb{L}^{p}(p>2)$-strong convergence. Therefore, based on the above discussion, an attempt will be made to establish an averaging principle for two-time-scales SPDEs driven by jumps in $\mathbb{L}^{p}(p>2)$-strong convergence. In this paper, our key and novelty is how to cope with the changes caused by jumps and higher order moments. It is drastically different because of the appearance of the jumps.

The paper is organized as follows. In Section 2, we present some notations and the formulation of the problem. In Section 3, the main result is stated. We derive the stochastic averaging principle for two-time-scales SPDEs driven by jumps in $\mathbb{L}^{p}(p>2)$-strong convergence.

\section{Preliminaries}

Let $(\Omega, \mathcal{F}, \mathbb{P})$ be a complete probability space with a natural filtration $\left\{\mathcal{F}_{t}\right\}_{t \geq 0}$ satisfying the usual conditions. We fix $l>0$ arbitrarily, and we denote $D:=(0, l)$, i.e., $D$ is a fixed, open, bounded interval of the real line $\mathbb{R}$. Let $\mathbb{H}$ be a Hilbert space $\mathbb{L}^{2}(D)$ equipped with the inner product $\langle\cdot, \cdot\rangle_{\mathbb{H}}$ and the corresponding norm $\|\cdot\|$. Let $T>0$ be fixed arbitrarily. In this paper, we are concerned with the following SPDEs driven by both Brownian motions 
and Poisson random measures:

$$
\left\{\begin{aligned}
\frac{\partial X_{t}^{\varepsilon}(\xi)}{\partial t}= & \Delta X_{t}^{\varepsilon}(\xi)+f\left(X_{t}^{\varepsilon}(\xi), Y_{t}^{\varepsilon}(\xi)\right)+g\left(X_{t}^{\varepsilon}(\xi)\right) \dot{W}_{t}^{1} \\
& +\int_{\mathbb{Z}} h\left(X_{t-}^{\varepsilon}(\xi), z\right) \tilde{\tilde{N}}_{1}(t, d z) \\
\frac{\partial Y_{t}^{\varepsilon}(\xi)}{\partial t}= & \frac{1}{\varepsilon} \Delta Y_{t}^{\varepsilon}(\xi)+\frac{1}{\varepsilon} F\left(X_{t}^{\varepsilon}(\xi), Y_{t}^{\varepsilon}(\xi)\right)+\frac{1}{\sqrt{\varepsilon}} G\left(X_{t}^{\varepsilon}(\xi), Y_{t}^{\varepsilon}(\xi)\right) \dot{W}_{t}^{2} \\
& +\int_{\mathbb{Z}} H\left(X_{t-}^{\varepsilon}(\xi), Y_{t-}^{\varepsilon}(\xi), z\right) \dot{\tilde{N}}_{2}^{\varepsilon}(t, d z) \\
X_{t}^{\varepsilon}(\xi)= & Y_{t}^{\varepsilon}(\xi)=0, \quad(\xi, t) \in \partial D \times(0, T], \\
X_{0}^{\varepsilon}(\xi)= & X_{0}(\xi) \in \mathbb{H}, \quad Y_{0}^{\varepsilon}(\xi)=Y_{0}(\xi) \in \mathbb{H}, \quad \xi \in D,
\end{aligned}\right.
$$

for $\varepsilon>0$ and $(\xi, t) \in D \times[0, T]$, where the coefficients $f(x, y): \mathbb{R} \times \mathbb{R} \rightarrow \mathbb{R}, g(x): \mathbb{R} \rightarrow \mathbb{R}$, $h(x, z): \mathbb{R} \times \mathbb{Z} \rightarrow \mathbb{R}, F(x, y): \mathbb{R} \times \mathbb{R} \rightarrow \mathbb{R}, G(x, y): \mathbb{R} \times \mathbb{R} \rightarrow \mathbb{R}, H(x, y, z): \mathbb{R} \times \mathbb{R} \times \mathbb{Z} \rightarrow \mathbb{R}$ are all real-valued measurable functions. The detailed conditions for them will be given in the next section. $\left\{W_{t}^{1}\right\}_{t \geq 0}$ and $\left\{W_{t}^{2}\right\}_{t \geq 0}$ are mutually independent real-valued $\left\{\mathcal{F}_{t}\right\}_{t \geq 0}$-Wiener processes. Next, we explicate the Poisson random measures $\tilde{N}_{1}(d t, d z)$ and $\tilde{N}_{2}^{\varepsilon}(d t, d z)$. Let $(\mathbb{Z}, \mathcal{B}(\mathbb{Z}))$ be a given measurable space and $v(d z)$ be a $\sigma$-finite measure on it. Let $D_{p_{t}^{i}}, i=1,2$ be two countable subsets of $\mathbb{R}_{+}$. Furthermore, let $p_{t}^{1}, t \in D_{p_{t}^{1}}$ be a stationary $\mathcal{F}_{t}$-adapted Poisson point process on $\mathbb{Z}$ with characteristic $v$, and let $p_{t}^{2}, t \in D_{p_{t}^{2}}$ be a stationary $\mathcal{F}_{t^{-}}$ adapted Poisson point process on $\mathbb{Z}$ with characteristic $\frac{v}{\varepsilon}$. Denote by $N^{i}(d t, d z)$ the Poisson counting measure associated with $p_{t}^{i}$, i.e.,

$$
N^{i}(t, A):=\sum_{s \in D_{p_{t}^{i}, s \leq t}} I_{A}\left(p_{t}^{i}\right), \quad i=1,2 .
$$

Let us denote the two corresponding compensated martingale measures

$$
\tilde{N}_{1}(d t, d z):=N^{1}(d t, d z)-v(d z) d t
$$

and

$$
\tilde{N}_{2}^{\varepsilon}(d t, d z):=N^{2}(d t, d z)-\frac{1}{\varepsilon} v(d z) d t .
$$

Let us define an abstract $\mathbb{A}=\partial_{\xi \xi}$ with zero Dirichlet boundary conditions. Let $\left\{e_{k}(\xi)\right\}_{k \in \mathbb{N}}$ be a complete orthonormal system of eigenvectors in $\mathbb{H}$ such that, for $k=1,2, \ldots$,

$$
\mathbb{A} e_{k}=-\alpha_{k} e_{k},\left.\quad e_{k}\right|_{\partial D=0},
$$

with $0<\alpha_{1} \leq \alpha_{2} \leq \cdots \leq \alpha_{k} \leq \cdots$.

Let $\mathbb{V}$ be a Sobolev space $H_{0}^{1}$ of order one with zero Dirichlet boundary conditions, which is densely and continuously injected in the Hilbert space $\mathbb{H}$. Identifying $\mathbb{H}$ with its dual space, we obtain the Gelfand triple

$$
\mathbb{V} \subset \mathbb{H} \cong \mathbb{H}^{*} \subset \mathbb{V}^{*}
$$

Owing to Poincarés inequality, we obtain

$$
\langle\mathbb{A} u, u\rangle=-\|\nabla u\|^{2} \leq-\alpha_{1}\|u\|^{2},
$$

where $\langle\cdot, \cdot\rangle$ denotes a dual pair of $\left(\mathbb{V}, \mathbb{V}^{*}\right)$. 
Note that the Green's function $S(\xi, \zeta, t)$ for the deterministic equation $(\partial / \partial t-\mathbb{A}) X(t, \xi)=$ 0 can be expressed as

$$
S(\xi, \zeta, t)=\sum_{k=1}^{\infty} e^{-\alpha_{k} t} e_{k}(\xi) e_{k}(\zeta)
$$

Recall that the associated Green's operator is defined, for any $\Lambda(\xi) \in \mathbb{H}$, by

$$
S_{t} \Lambda(\xi)=\int_{D} S(\xi, \zeta, t) \Lambda(\zeta) d \zeta=\sum_{k=1}^{\infty} e^{-\alpha_{k} t} e_{k}(\xi)\left\langle e_{k}, \Lambda\right\rangle_{\mathbb{H}}
$$

It is straightforward that $\left\{S_{t}\right\}_{t \geq 0}$ are contractive semigroups on $\mathbb{H}$ and $\left\|S_{t} \Lambda(\xi)\right\| \leq\|\Lambda(\xi)\|$.

To give precise results, it is convenient to look at the equations in an abstract setting, where system (2.1) can be rewritten as

$$
\left\{\begin{aligned}
& d X_{t}^{\varepsilon}= {\left[\mathbb{A} X_{t}^{\varepsilon}+f\left(X_{t}^{\varepsilon}, Y_{t}^{\varepsilon}\right)\right] d t+g\left(X_{t}^{\varepsilon}\right) d W_{t}^{1}+\int_{\mathbb{Z}} h\left(X_{t-}^{\varepsilon}, z\right) \tilde{N}_{1}(d t, d z) } \\
& d Y_{t}^{\varepsilon}= \frac{1}{\varepsilon}\left[\mathbb{A} Y_{t}^{\varepsilon}+F\left(X_{t}^{\varepsilon}, Y_{t}^{\varepsilon}\right)\right] d t+\frac{1}{\sqrt{\varepsilon}} G\left(X_{t}^{\varepsilon}, Y_{t}^{\varepsilon}\right) d W_{t}^{2} \\
&+\int_{\mathbb{Z}} H\left(X_{t-}^{\varepsilon}, Y_{t-}^{\varepsilon}, z\right) \tilde{N}_{2}^{\varepsilon}(d t, d z) \\
& X_{0} \in \mathbb{H}, \quad Y_{0} \in \mathbb{H} .
\end{aligned}\right.
$$

We now introduce the definition of mild solutions of system (2.3).

Definition 2.1 A natural way to give a rigorous meaning to (2.3) is in terms of the following integral equations:

$$
\left\{\begin{aligned}
X_{t}^{\varepsilon}= & X_{0} S_{t}+\int_{0}^{t} S_{t-s} f\left(X_{s}^{\varepsilon}, Y_{s}^{\varepsilon}\right) d s+\int_{0}^{t} S_{t-s} g\left(X_{s}^{\varepsilon}\right) d W_{s}^{1} \\
& +\int_{0}^{t} \int_{\mathbb{Z}} S_{t-s} h\left(X_{s-}^{\varepsilon}, z\right) \tilde{N}_{1}(d s, d z), \\
Y_{t}^{\varepsilon}= & Y_{0} S_{t / \varepsilon}+\frac{1}{\varepsilon} \int_{0}^{t} S_{(t-s) / \varepsilon} F\left(X_{s}^{\varepsilon}, Y_{s}^{\varepsilon}\right) d s+\frac{1}{\sqrt{\varepsilon}} \int_{0}^{t} S_{(t-s) / \varepsilon} G\left(X_{s}^{\varepsilon}, Y_{s}^{\varepsilon}\right) d W_{s}^{2} \\
& +\int_{0}^{t} \int_{\mathbb{Z}} S_{(t-s) / \varepsilon} H\left(X_{s-}^{\varepsilon}, Y_{s-}^{\varepsilon}, z\right) \tilde{N}_{2}^{\varepsilon}(d s, d z) .
\end{aligned}\right.
$$

Moreover, according to Itô's formula $[39,47]$, for $t \in[0, T], p>1$, the following equalities hold:

$$
\begin{aligned}
\left\|X_{t}^{\epsilon}\right\|^{2 p}= & \left\|X_{0}\right\|^{2 p}+2 p \int_{0}^{t}\left\|X_{s}^{\epsilon}\right\|^{2 p-2}\left\langle\mathbb{A} X_{s}^{\epsilon}, X_{s}^{\epsilon}\right\rangle d s \\
& +2 p \int_{0}^{t}\left\|X_{s}^{\epsilon}\right\|^{2 p-2}\left\langle f\left(X_{s}^{\epsilon}, Y_{s}^{\epsilon}\right), X_{s}^{\epsilon}\right\rangle_{\mathbb{H}} d s \\
& +2 p \int_{0}^{t}\left\|X_{s}^{\epsilon}\right\|^{2 p-2}\left\langle g\left(X_{s}^{\epsilon}\right), X_{s}^{\epsilon}\right\rangle_{\mathbb{H}} d W_{s}^{1} \\
& +2 p(p-1) \int_{0}^{t}\left\|X_{s}^{\epsilon}\right\|^{2 p-2}\left\|g\left(X_{s}^{\epsilon}\right)\right\|^{2} d s \\
& +\int_{0}^{t} \int_{\mathbb{Z}}\left[\left\|X_{s-}^{\epsilon}+h\left(X_{s-}^{\epsilon}, z\right)\right\|^{2 p}-\left\|X_{s-}^{\epsilon}\right\|^{2 p}\right] \tilde{N}_{1}(d s, d z) \\
& +\int_{0}^{t} \int_{\mathbb{Z}}\left[\left\|X_{s}^{\epsilon}+h\left(X_{s}^{\epsilon}, z\right)\right\|^{2 p}-\left\|X_{s}^{\epsilon}\right\|^{2 p}\right] v(d z) d s \\
& -2 p \int_{0}^{t} \int_{\mathbb{Z}}\left\|X_{s}^{\epsilon}\right\|^{2 p-2}\left\langle h\left(X_{s}^{\epsilon}, z\right), X_{s}^{\epsilon}\right\rangle_{\mathbb{H}} v(d z) d s
\end{aligned}
$$


and

$$
\begin{aligned}
\left\|Y_{t}^{\epsilon}\right\|^{2 p}= & \left\|Y_{0}\right\|^{2 p}+\frac{2 p}{\epsilon} \int_{0}^{t}\left\|Y_{s}^{\epsilon}\right\|^{2 p-2}\left\langle\mathbb{A} Y_{s}^{\epsilon}, Y_{s}^{\epsilon}\right\rangle d s \\
& +\frac{2 p}{\epsilon} \int_{0}^{t}\left\|Y_{s}^{\epsilon}\right\|^{2 p-2}\left\langle F\left(X_{s}^{\epsilon}, Y_{s}^{\epsilon}\right), Y_{s}^{\epsilon}\right\rangle_{\mathbb{H}} d s \\
& +\frac{2 p}{\sqrt{\epsilon}} \int_{0}^{t}\left\|Y_{s}^{\epsilon}\right\|^{2 p-2}\left\langle G\left(X_{s}^{\epsilon}, Y_{s}^{\epsilon}\right), Y_{s}^{\epsilon}\right\rangle_{\mathbb{H}} d W_{s}^{2} \\
& +\frac{2 p(p-1)}{\epsilon} \int_{0}^{t}\left\|Y_{s}^{\epsilon}\right\|^{2 p-2}\left\|G\left(X_{s}^{\epsilon}, Y_{s}^{\epsilon}\right)\right\|^{2} d s \\
& +\int_{0}^{t} \int_{\mathbb{Z}}\left[\left\|Y_{s-}^{\epsilon}+H\left(X_{s-}^{\epsilon}, Y_{s-}^{\epsilon}, z\right)\right\|^{2 p}-\left\|Y_{s-}^{\epsilon}\right\|^{2 p}\right] \tilde{N}_{2}^{\epsilon}(d s, d z) \\
& +\frac{1}{\epsilon} \int_{0}^{t} \int_{\mathbb{Z}}\left[\left\|Y_{s}^{\epsilon}+H\left(X_{s}^{\epsilon}, Y_{s}^{\epsilon}, z\right)\right\|^{2 p}-\left\|Y_{s}^{\epsilon}\right\|^{2 p}\right] v(d z) d s \\
& -\frac{2 p}{\epsilon} \int_{0}^{t} \int_{\mathbb{Z}}\left\|Y_{s}^{\epsilon}\right\|^{2 p-2}\left\langle H\left(X_{s}^{\epsilon}, Y_{s}^{\epsilon}, z\right), Y_{s}^{\epsilon}\right\rangle_{\mathbb{H}} v(d z) d s .
\end{aligned}
$$

Convention The letter $C$, with or without subscripts, will denote positive constants whose value may change in different occasions. We will write the dependence of constants on parameters explicitly if it is essential.

Now, we need to give some dissipative conditions [46] to ensure the ergodicity for the fast motion and global Lipschitz condition, and the growth condition to ensure the existence and uniqueness for (2.3).

Assumption 1 The coefficients of (2.3) are globally Lipschitz continuous in $x, y$, i.e., $\forall x_{1}, x_{2}, y_{1}, y_{2} \in \mathbb{R}$, there exist six positive constants $C_{f}, C_{g}, C_{h}, C_{F}, C_{G}, C_{H}$. We have

$$
\begin{aligned}
& \left|f\left(x_{1}, y_{1}\right)-f\left(x_{2}, y_{2}\right)\right|^{2} \leq C_{f}\left(\left|x_{1}-x_{2}\right|^{2}+\left|y_{1}-y_{2}\right|^{2}\right), \\
& \left|g\left(x_{1}\right)-g\left(x_{2}\right)\right|^{2} \leq C_{g}\left|x_{1}-x_{2}\right|^{2}, \\
& \int_{\mathbb{Z}}\left|h\left(x_{1}, z\right)-h\left(x_{2}, z\right)\right|^{q} v(d z) \leq C_{h}\left|x_{1}-x_{2}\right|^{q}, \quad q \geq 2,
\end{aligned}
$$

and

$$
\begin{aligned}
& \left|F\left(x_{1}, y_{1}\right)-F\left(x_{2}, y_{2}\right)\right|^{2} \leq C_{F}\left(\left|x_{1}-x_{2}\right|^{2}+\left|y_{1}-y_{2}\right|^{2}\right) \\
& \left|G\left(x_{1}, y_{1}\right)-G\left(x_{2}, y_{2}\right)\right|^{2} \leq C_{G}\left(\left|x_{1}-x_{2}\right|^{2}+\left|y_{1}-y_{2}\right|^{2}\right), \\
& \int_{\mathbb{Z}}\left|H\left(x_{1}, y_{1}, z\right)-H\left(x_{2}, y_{2}, z\right)\right|^{q} v(d z) \leq C_{H}\left(\left|x_{1}-x_{2}\right|^{q}+\left|y_{1}-y_{2}\right|^{q}\right), \quad q \geq 2 .
\end{aligned}
$$

Remark 2.2 From Assumption 1, for all $x_{1}, y_{1} \in \mathbb{R}$, it immediately follows that

$$
\begin{aligned}
& \left\|f\left(x_{1}, y_{1}\right)\right\|^{2}+\left\|g\left(x_{1}\right)\right\|^{2}+\left\|F\left(x_{1}, y_{1}\right)\right\|^{2}+\left\|G\left(x_{1}, y_{1}\right)\right\|^{2} \\
& \leq 2\left(C_{f}+C_{g}+C_{F}+C_{G}\right)\left(\left\|x_{1}\right\|^{2}+\left\|y_{1}\right\|^{2}\right) \\
& \quad+2\left(|f(0,0)|^{2}+|g(0)|^{2}+|F(0,0)|^{2}+|G(0,0)|^{2}\right),
\end{aligned}
$$




$$
\begin{aligned}
& \int_{\mathbb{Z}}\left\|h\left(x_{1}, z\right)\right\|^{q} v(d z) \leq 2^{q-1} \int_{\mathbb{Z}}\|h(0, z)\|^{q} v(d z)+2^{q-1} C_{h}\left\|x_{1}\right\|^{q}, \\
& \int_{\mathbb{Z}}\left\|H\left(x_{1}, y_{1}, z\right)\right\|^{q} v(d z) \leq 2^{q-1} \int_{\mathbb{Z}}\|H(0,0, z)\|^{q} v(d z)+2^{q-1} C_{H}\left(\left\|x_{1}\right\|^{q}+\left\|y_{1}\right\|^{q}\right), \quad q \geq 2,
\end{aligned}
$$

so we set

$$
\begin{aligned}
& K_{3}=\max \left\{2\left(C_{f}+C_{g}+C_{F}+C_{G}\right), 2\left(|f(0,0)|^{2}+|g(0)|^{2}+|F(0,0)|^{2}+|G(0,0)|^{2}\right)\right\}, \\
& K_{4}=\max \left\{2^{q-1} \int_{\mathbb{Z}}\|h(0, z)\|^{q} v(d z), 2^{q-1} C_{h}\right\}, \\
& K_{5}=\max \left\{2^{q-1} \int_{\mathbb{Z}}\|H(0,0, z)\|^{q} v(d z), 2^{q-1} C_{H}\right\},
\end{aligned}
$$

and then we have

$$
\begin{aligned}
& \left\|f\left(x_{1}, y_{1}\right)\right\|^{2}+\left\|g\left(x_{1}\right)\right\|^{2}+\left\|F\left(x_{1}, y_{1}\right)\right\|^{2}+\left\|G\left(x_{1}, y_{1}\right)\right\|^{2} \leq K_{3}\left(1+\left\|x_{1}\right\|^{2}+\left\|y_{1}\right\|^{2}\right), \\
& \int_{\mathbb{Z}}\left\|h\left(x_{1}, z\right)\right\|^{q} v(d z) \leq K_{4}\left(1+\left\|x_{1}\right\|^{q}\right), \quad q \geq 2, \\
& \int_{\mathbb{Z}}\left\|H\left(x_{1}, y_{1}, z\right)\right\|^{q} v(d z) \leq K_{5}\left(1+\left\|x_{1}\right\|^{q}+\left\|y_{1}\right\|^{q}\right), \quad q \geq 2 .
\end{aligned}
$$

Assumption $2 f$ is globally bounded.

Assumption $3 \eta=\alpha_{1}-C_{F}-C_{G}-C_{H}>0$, where $\alpha_{1}$ is the decay rate of $\mathbb{A}$.

Remark 2.3 Assumption 3 is a strong dissipative condition, and it is very important to prove the ergodicity for the fast motion. The detailed proofs will be given in Appendix A.

It is easy to see that, complying with Assumption 1, and in terms of Remark 2.3, (2.3) has unique mild solutions $[39,40]$.

\section{Averaging principle for two-time-scales SPDEs with jumps}

In this section, we first prove two key lemmas and then present the main result of the paper.

Lemma 3.1 Let Assumptions 1-3 be satisfied. For any $T>0$ there exists a positive constant $C_{p, T}>0, p>1$ such that for any $\epsilon \in(0,1)$,

$$
\mathbb{E} \sup _{0 \leq t \leq T}\left\|X_{t}^{\epsilon}\right\|^{2 p} \leq C_{p, T} .
$$

Proof For $\left\|X_{t}^{\epsilon}\right\|^{2 p}$, by the energy identities (2.5), we have

$$
\begin{aligned}
\left\|X_{t}^{\epsilon}\right\|^{2 p}= & \left\|X_{0}\right\|^{2 p}+2 p \int_{0}^{t}\left\|X_{s}^{\epsilon}\right\|^{2 p-2}\left\langle\mathbb{A} X_{s}^{\epsilon}, X_{s}^{\epsilon}\right\rangle d s \\
& +2 p \int_{0}^{t}\left\|X_{s}^{\epsilon}\right\|^{2 p-2}\left\langle f\left(X_{s}^{\epsilon}, Y_{s}^{\epsilon}\right), X_{s}^{\epsilon}\right\rangle_{\mathbb{H}} d s \\
& +2 p \int_{0}^{t}\left\|X_{s}^{\epsilon}\right\|^{2 p-2}\left\langle g\left(X_{s}^{\epsilon}\right), X_{s}^{\epsilon}\right\rangle_{\mathbb{H}} d W_{s}^{1}
\end{aligned}
$$




$$
\begin{aligned}
& +2 p(p-1) \int_{0}^{t}\left\|X_{s}^{\epsilon}\right\|^{2 p-2}\left\|g\left(X_{s}^{\epsilon}\right)\right\|^{2} d s \\
& +\int_{0}^{t} \int_{\mathbb{Z}}\left[\left\|X_{s-}^{\epsilon}+h\left(X_{s-}^{\epsilon}, z\right)\right\|^{2 p}-\left\|X_{s-}^{\epsilon}\right\|^{2 p}\right] \tilde{N}_{1}(d s, d z) \\
& +\int_{0}^{t} \int_{\mathbb{Z}}\left[\left\|X_{s}^{\epsilon}+h\left(X_{s}^{\epsilon}, z\right)\right\|^{2 p}-\left\|X_{s}^{\epsilon}\right\|^{2 p}\right] v(d z) d s \\
& -2 p \int_{0}^{t} \int_{\mathbb{Z}}\left\|X_{s}^{\epsilon}\right\|^{2 p-2}\left\langle h\left(X_{s}^{\epsilon}, z\right), X_{s}^{\epsilon}\right\rangle_{\mathbb{H}} v(d z) d s \\
& =\left\|X_{0}\right\|^{2 p}+\sum_{i=1}^{7} \Pi_{t}^{i} .
\end{aligned}
$$

By Assumption 1 and Assumption 3, Young's inequality and (2.2), we have

$$
\begin{aligned}
\left\|X_{0}\right\|^{2 p}+\Pi_{t}^{1}+\Pi_{t}^{2}+\Pi_{t}^{4} & \leq\left\|X_{0}\right\|^{2 p}-2 \alpha_{1} p \int_{0}^{t}\left\|X_{s}^{\epsilon}\right\|^{2 p} d s+C_{p} \int_{0}^{t}\left\|X_{s}^{\epsilon}\right\|^{2 p} d s \\
& \leq C+C_{p} \int_{0}^{t}\left\|X_{s}^{\epsilon}\right\|^{2 p} d s .
\end{aligned}
$$

For $\Pi_{t}^{6}, \Pi_{t}^{7}$, according to the binomial theorem, we calculate the coefficients in the expansion of $(a+b)^{2 p}$,

$$
\begin{aligned}
(a+b)^{2 p}= & C_{0}^{2 p} a^{2 p}+C_{1}^{2 p} a^{2 p-1} b+C_{2}^{2 p} a^{2 p-2} b^{2} \\
& +\cdots+C_{2 p-2}^{2 p} a^{2} b^{2 p-2}+C_{2 p-1}^{2 p} a b^{2 p-1}+C_{2 p}^{2 p} b^{2 p}
\end{aligned}
$$

where $C_{k}^{2 p}=\frac{(2 p) !}{(2 p-k) ! k !}, k=0,1,2, \ldots, 2 p$. So, by Assumption 3 and Young's inequality,

$$
\begin{aligned}
\Pi_{t}^{6}+\Pi_{t}^{7}= & \int_{0}^{t} \int_{\mathbb{Z}}\left[\left\|X_{s}^{\epsilon}+h\left(X_{s}^{\epsilon}, z\right)\right\|^{2 p}-\left\|X_{s}^{\epsilon}\right\|^{2 p}\right] v(d z) d s \\
& -2 p \int_{0}^{t} \int_{\mathbb{Z}}\left\|X_{s}^{\epsilon}\right\|^{2 p-2}\left\langle h\left(X_{s}^{\epsilon}, z\right), X_{s}^{\epsilon}\right\rangle_{\mathbb{H}} v(d z) d s \\
= & \sum_{i=2}^{2 p} C_{i}^{2 p} \int_{0}^{t} \int_{\mathbb{Z}}\left\|X_{s}^{\epsilon}\right\|^{2 p-i}\left\|h\left(X_{s}^{\epsilon}, z\right)\right\|^{i} v(d z) d s \\
\leq & C_{p} \int_{0}^{t}\left\|X_{s}^{\epsilon}\right\|^{2 p} d s+C .
\end{aligned}
$$

Then we obtain

$$
\mathbb{E} \sup _{0 \leq s \leq t}\left\|X_{t}^{\epsilon}\right\|^{2 p} \leq C+C_{p} \int_{0}^{t} \mathbb{E} \sup _{0 \leq r \leq s}\left\|X_{r}^{\epsilon}\right\|^{2 p} d s+\mathbb{E} \sup _{0 \leq s \leq t} \Pi_{s}^{3}+\mathbb{E} \sup _{0 \leq s \leq t} \Pi_{s}^{5} .
$$

Now, by Young's inequality and the BDG inequality, we find

$$
\begin{aligned}
\mathbb{E} \sup _{0 \leq s \leq t} \Pi_{s}^{5} & \leq C \mathbb{E}\left\{\int_{0}^{t} \int_{\mathbb{Z}}\left[\left\|X_{s}^{\epsilon}+h\left(X_{s}^{\epsilon}, z\right)\right\|^{2 p}-\left\|X_{s}^{\epsilon}\right\|^{2 p}\right]^{2} v(d z) d s\right\}^{\frac{1}{2}} \\
& \leq C \mathbb{E}\left\{\int_{0}^{t} \int_{\mathbb{Z}}\left(\sum_{i=1}^{2 p} C_{i}^{2 p}\left\|X_{s}^{\epsilon}\right\|^{2 p-i}\left\|h\left(X_{s}^{\epsilon}, z\right)\right\|^{i}\right)^{2} v(d z) d s\right\}^{\frac{1}{2}}
\end{aligned}
$$




$$
\begin{aligned}
& \leq C \mathbb{E}\left\{\sum_{i=1}^{2 p} \int_{0}^{t} \int_{\mathbb{Z}}\left\|X_{s}^{\epsilon}\right\|^{4 p-2 i}\left\|h\left(X_{s}^{\epsilon}, z\right)\right\|^{2 i} v(d z) d s\right\}^{\frac{1}{2}} \\
& \leq C \mathbb{E}\left\{\int_{0}^{t}\left\|X_{s}^{\epsilon}\right\|^{4 p} d s\right\}^{\frac{1}{2}}+C .
\end{aligned}
$$

Next, it is easy to see that

$$
\mathbb{E} \sup _{0 \leq s \leq t} \Pi_{s}^{3} \leq C \mathbb{E}\left\{\int_{0}^{t}\left\|X_{s}^{\epsilon}\right\|^{4 p} d s\right\}^{\frac{1}{2}}+C .
$$

Therefore,

$$
\begin{aligned}
\mathbb{E} \sup _{0 \leq s \leq t}\left\|X_{s}^{\epsilon}\right\|^{2 p} & \leq C+C_{p} \int_{0}^{t} \mathbb{E} \sup _{0 \leq r \leq s}\left\|X_{r}^{\epsilon}\right\|^{2 p} d s+C \mathbb{E}\left\{\int_{0}^{t}\left\|X_{s}^{\epsilon}\right\|^{4 p} d s\right\}^{\frac{1}{2}} \\
& \leq C+C_{p} \int_{0}^{t} \mathbb{E} \sup _{0 \leq r \leq s}\left\|X_{r}^{\epsilon}\right\|^{2 p} d s+C \mathbb{E}\left\{\sup _{0 \leq s \leq t}\left\|X_{s}^{\epsilon}\right\|^{2 p} \int_{0}^{t}\left\|X_{s}^{\epsilon}\right\|^{2 p} d s\right\}^{\frac{1}{2}} \\
& \leq C+C_{p} \int_{0}^{t} \mathbb{E} \sup _{0 \leq r \leq s}\left\|X_{r}^{\epsilon}\right\|^{2 p} d s+\frac{1}{2} \mathbb{E} \sup _{0 \leq s \leq t}\left\|X_{s}^{\epsilon}\right\|^{2 p}
\end{aligned}
$$

Finally, by Gronwall's inequality, we have

$$
\mathbb{E} \sup _{0 \leq s \leq t}\left\|X_{s}^{\epsilon}\right\|^{2 p} \leq C e^{C_{p} T}
$$

This is the proof of Lemma 3.1.

Lemma 3.2 Let Assumptions 1-3 be satisfied. For any $T>0$, there exists a positive constant $C_{p, \alpha_{1}, C_{F}, K_{3}, K_{5}, \gamma}>0$ such that for any $\epsilon \in(0,1), \gamma>0$,

$$
\sup _{0 \leq t \leq T} \mathbb{E}\left\|Y_{t}^{\epsilon}\right\|^{2 p} \leq C_{p, \alpha_{1}, C_{F}, K_{3}, K_{5}, \gamma}
$$

Proof Due to the energy identity (2.6), we find

$$
\begin{aligned}
\mathbb{E}\left\|Y_{t}^{\epsilon}\right\|^{2 p}= & \left\|Y_{0}\right\|^{2 p}+\frac{2 p}{\epsilon} \mathbb{E} \int_{0}^{t}\left\|Y_{s}^{\epsilon}\right\|^{2 p-2}\left\langle\mathbb{A} Y_{s}^{\epsilon}, Y_{s}^{\epsilon}\right\rangle d s \\
& +\frac{2 p}{\epsilon} \mathbb{E} \int_{0}^{t}\left\|Y_{s}^{\epsilon}\right\|^{2 p-2}\left\langle F\left(X_{s}^{\epsilon}, Y_{s}^{\epsilon}\right), Y_{s}^{\epsilon}\right\rangle_{\mathbb{H}} d s \\
& +\frac{2 p(p-1)}{\epsilon} \mathbb{E} \int_{0}^{t}\left\|Y_{s}^{\epsilon}\right\|^{2 p-2}\left\|G\left(X_{s}^{\epsilon}, Y_{s}^{\epsilon}\right)\right\|^{2} d s \\
& +\frac{1}{\epsilon} \mathbb{E} \int_{0}^{t} \int_{\mathbb{Z}}\left[\left\|Y_{s}^{\epsilon}+H\left(X_{s}^{\epsilon}, Y_{s}^{\epsilon}, z\right)\right\|^{2 p}-\left\|Y_{s}^{\epsilon}\right\|^{2 p}\right] v(d z) d s \\
& -\frac{2 p}{\epsilon} \mathbb{E} \int_{0}^{t} \int_{\mathbb{Z}}\left\|Y_{s}^{\epsilon}\right\|^{2 p-2}\left\langle H\left(X_{s}^{\epsilon}, Y_{s}^{\epsilon}, z\right), Y_{s}^{\epsilon}\right\rangle_{\mathbb{H}} \nu(d z) d s \\
= & \left\|Y_{0}\right\|^{2 p}+\sum_{i=1}^{5} \Xi_{t}^{i} .
\end{aligned}
$$


In view of Assumption 1 and Assumption 3, we have

$$
\begin{aligned}
& \left\langle\mathbb{A} Y_{s}^{\epsilon}, Y_{s}^{\epsilon}\right\rangle \leq-\alpha_{1}\left\|Y_{s}^{\epsilon}\right\|^{2}, \\
& \left\langle F\left(X_{s}^{\epsilon}, Y_{s}^{\epsilon}\right), Y_{s}^{\epsilon}\right\rangle_{\mathbb{H}}=\left\langle F\left(X_{s}^{\epsilon}, Y_{s}^{\epsilon}\right)-F\left(X_{s}^{\epsilon}, 0\right), Y_{s}^{\epsilon}\right\rangle_{\mathbb{H}}+\left\langle F\left(X_{s}^{\epsilon}, 0\right), Y_{s}^{\epsilon}\right\rangle_{\mathbb{H}}, \\
& \left\|G\left(X_{s}^{\epsilon}, Y_{s}^{\epsilon}\right)\right\|^{2} \leq K_{3}\left(1+\left\|Y_{s}^{\epsilon}\right\|^{2}+\left\|X_{s}^{\epsilon}\right\|^{2}\right) .
\end{aligned}
$$

Then by taking (3.2) and $\gamma>0$ small enough for Young's inequality in the form $|a b| \leq$ $\gamma|b|^{m}+C_{r, m}|a|^{\frac{m}{m-1}}$, we have

$$
\begin{aligned}
\left\|Y_{0}\right\|^{2 p}+\sum_{i=1}^{3} \Xi_{t}^{i} \leq & \left\|Y_{0}\right\|^{2 p}-\frac{2 p \alpha_{1}}{\epsilon} \mathbb{E} \int_{0}^{t}\left\|Y_{s}^{\epsilon}\right\|^{2 p} d s+\frac{p\left(C_{F}+1\right)}{\epsilon} \mathbb{E} \int_{0}^{t}\left\|Y_{s}^{\epsilon}\right\|^{2 p} d s \\
& +\frac{p}{\epsilon} \mathbb{E} \int_{0}^{t}\left\|Y_{s}^{\epsilon}\right\|^{2 p} d s+\frac{p}{\epsilon} \mathbb{E} \int_{0}^{t}\left\|Y_{s}^{\epsilon}\right\|^{2 p-2} C_{F}\left(1+\left\|X_{s}^{\epsilon}\right\|^{2}\right) d s \\
& +\frac{2 p(p-1)}{\epsilon} K_{3} \mathbb{E} \int_{0}^{t}\left\|Y_{s}^{\epsilon}\right\|^{2 p-2}\left(1+\left\|Y_{s}^{\epsilon}\right\|^{2}+\left\|X_{s}^{\epsilon}\right\|^{2}\right) d s \\
\leq & \left\|Y_{0}\right\|^{2 p}-\frac{C_{p, \alpha_{1}, C_{F}, K_{3}, \gamma}}{\epsilon} \mathbb{E} \int_{0}^{t}\left\|Y_{s}^{\epsilon}\right\|^{2 p} d s \\
& +\frac{C_{p}^{\prime}}{\epsilon} \mathbb{E} \int_{0}^{t}\left\|X_{s}^{\epsilon}\right\|^{2 p} d s+\frac{C_{p}^{\prime} t}{\epsilon} .
\end{aligned}
$$

By the binomial theorem (3.1), Young's inequality and Assumption 3, we have

$$
\begin{aligned}
\Xi_{t}^{4}+\Xi_{t}^{5} & \leq \frac{1}{\epsilon} \sum_{i=2}^{2 p} C_{i}^{2 p} \mathbb{E} \int_{0}^{t} \int_{\mathbb{Z}}\left\|Y_{s}^{\epsilon}\right\|^{2 p-i}\left\|H\left(X_{s}^{\epsilon}, Y_{s}^{\epsilon}, z\right)\right\|^{i} v(d z) d s \\
& \leq \frac{1}{\epsilon} K_{5} \sum_{i=2}^{2 p} C_{i}^{2 p} \mathbb{E} \int_{0}^{t}\left\|Y_{s}^{\epsilon}\right\|^{2 p-i}\left(1+\left\|X_{s}^{\epsilon}\right\|^{i}+\left\|Y_{s}^{\epsilon}\right\|^{i}\right) d s \\
& \leq \frac{C_{p, K_{5}, \gamma}}{\epsilon} \mathbb{E} \int_{0}^{t}\left\|Y_{s}^{\epsilon}\right\|^{2 p} d s+\frac{C_{p}^{\prime}}{\epsilon} \mathbb{E} \int_{0}^{t}\left\|X_{s}^{\epsilon}\right\|^{2 p} d s+\frac{C_{p}^{\prime} t}{\epsilon} .
\end{aligned}
$$

With the help of Gronwall's inequality (see reference [17], p.74), we know there exists a positive constant $C_{p, \alpha_{1}, C_{F}, K_{3}, K_{5}, \gamma}>0$. We have

$$
\begin{aligned}
\sup _{0 \leq s \leq T} \mathbb{E}\left\|Y_{s}^{\epsilon}\right\|^{2 p} & \leq\left\|Y_{0}\right\|^{2 p}-\frac{C_{p, \alpha_{1}, C_{F}, K_{3}, K_{5}, \gamma}}{\epsilon} \mathbb{E} \int_{0}^{t} \sup _{0 \leq r \leq s}\left\|Y_{r}^{\epsilon}\right\|^{2 p} d r+\frac{C_{p}^{\prime}}{\epsilon} t \\
& \leq\left\|Y_{0}\right\|^{2 p} e^{-\frac{C_{p, \alpha_{1}, C_{F}, K_{3}, K_{5}, \gamma}}{\epsilon}}+C_{p}^{\prime}\left(e^{-\frac{C_{p, \alpha_{1}, C_{F}, K_{3}, K_{5}, \gamma}}{\epsilon} T}-1\right) \\
& \leq C_{p, \alpha_{1}, C_{F}, K_{3}, K_{5}, \gamma} .
\end{aligned}
$$

This is the proof of Lemma 3.2.

Theorem 3.3 Let Assumptions 1-3 be satisfied. $\bar{X}_{t}$ denotes the stochastic process determined by the SPDE

$$
d \bar{X}_{t}=\left[\mathbb{A} \bar{X}_{t}+\bar{f}\left(\bar{X}_{t}\right)\right] d t+g\left(\bar{X}_{t}\right) d W_{t}^{1}+\int_{\mathbb{Z}} h\left(\bar{X}_{t-}, z\right) \tilde{N}_{1}(d t, d z)
$$


Then for $T>0, p>1$, we have

$$
\mathbb{E} \sup _{0 \leq t \leq T}\left\|X_{t}^{\epsilon}-\bar{X}_{t}\right\|^{2 p} \rightarrow 0
$$

as $\epsilon \rightarrow 0$.

Proof In order to prove the above theorem Theorem 3.3, we divide the course of the proof in three steps. In Step $1,\left\|X_{t}^{\epsilon}-\hat{X}_{t}^{\epsilon}\right\|^{2 p}$ will be estimated. We prove the other estimate $\left\|\hat{X}_{t}^{\epsilon}-\bar{X}_{t}\right\|^{2 p}$ in Step 2. Finally, through Step 1 and Step 2, Theorem 3.3 will be obtained.

Step 1. We consider a partition of $[0, T]$ into intervals of the same length $\delta(\delta<1)$. Then, for $t \in[k \delta, \min \{(k+1) \delta, T\}], k=0,1, \ldots,\lfloor T / \delta\rfloor$, we construct auxiliary processes $\hat{Y}_{t}^{\epsilon}$ and $\hat{X}_{t}^{\epsilon}$, by means of the relations

$$
\begin{aligned}
\hat{Y}_{t}^{\epsilon}= & Y_{k \delta}^{\epsilon}+\frac{1}{\epsilon} \int_{k \delta}^{t}\left[\mathbb{A} \hat{Y}_{s}^{\epsilon}+F\left(X_{k \delta}^{\epsilon}, \hat{Y}_{s}^{\epsilon}\right)\right] d s+\frac{1}{\sqrt{\epsilon}} \int_{k \delta}^{t} G\left(X_{k \delta}^{\epsilon}, \hat{Y}_{s}^{\epsilon}\right) d W_{s}^{2} \\
& +\int_{k \delta}^{t} \int_{\mathbb{Z}} H\left(X_{k \delta}^{\epsilon}, \hat{Y}_{s-}^{\epsilon}, z\right) \tilde{N}_{2}^{\epsilon}(d s, d z)
\end{aligned}
$$

and

$$
\begin{aligned}
\hat{X}_{t}^{\epsilon}= & X_{0}+\int_{0}^{t} \mathbb{A} X_{s}^{\epsilon} d s+\int_{0}^{t} f\left(X_{[s / \delta] \delta}^{\epsilon}, \hat{Y}_{s}^{\epsilon}\right) d s+\int_{0}^{t} g\left(X_{s}^{\epsilon}\right) d W_{s}^{1} \\
& +\int_{0}^{t} \int_{\mathbb{Z}} h\left(X_{s-}^{\epsilon}, z\right) \tilde{N}_{1}(d s, d z), \quad t \in[0, T] .
\end{aligned}
$$

To proceed, by the mild solution $X_{t}^{\epsilon}$ of (2.3), we make the following estimation:

$$
\begin{aligned}
\left\|X_{t}^{\epsilon}-X_{k \delta}^{\epsilon}\right\|^{2 p} \leq & 4^{2 p-1}\left\|X_{k \delta}^{\epsilon}\left(S_{t-k \delta}-\mathbb{I}\right)\right\|^{2 p}+4^{2 p-1}\left\|\int_{k \delta}^{t} S_{t-s} f\left(X_{s}^{\epsilon}, Y_{s}^{\epsilon}\right) d s\right\|^{2 p} \\
& +4^{2 p-1}\left\|\int_{k \delta}^{t} S_{t-s} g\left(X_{s}^{\epsilon}\right) d W_{s}^{1}\right\|^{2 p} \\
& +4^{2 p-1}\left\|\int_{k \delta}^{t} \int_{\mathbb{Z}} S_{t-s} h\left(X_{s-}^{\epsilon}, z\right) \tilde{N}_{1}(d s, d z)\right\|^{2 p} \\
= & I_{1}+I_{2}+I_{3}+I_{4},
\end{aligned}
$$

where II denotes the identity operator.

First of all, since $f$ is globally bounded, by Hölder's inequality and Assumption 3, detailed computation leads to

$$
\begin{aligned}
\mathbb{E} I_{2} & =4^{2 p-1}\left\|\int_{k \delta}^{t} S_{t-s} f\left(X_{s}^{\epsilon}, Y_{s}^{\epsilon}\right) d s\right\|^{2 p} \\
& \leq C\|t-k \delta\|^{2 p-1} \int_{k \delta}^{t}\left\|f\left(X_{s}^{\epsilon}, Y_{s}^{\epsilon}\right)\right\|^{2 p} d s \\
& \leq C\|t-k \delta\|^{2 p} .
\end{aligned}
$$


Second, from the BDG inequality, Hölder's inequality and Lemma 3.1, it follows that

$$
\begin{aligned}
\mathbb{E} I_{3} & =4^{2 p-1} \mathbb{E}\left[\int_{k \delta}^{t}\left\|S_{t-s} g\left(X_{s}^{\epsilon}\right)\right\|^{2} d s\right]^{p} \\
& \leq C\|t-k \delta\|^{p-1} \mathbb{E} \int_{k \delta}^{t}\left\|g\left(X_{s}^{\epsilon}\right)\right\|^{2 p} d s \\
& \leq C\|t-k \delta\|^{p-1} \int_{k \delta}^{t}\left(1+\mathbb{E}\left\|X_{s}^{\epsilon}\right\|^{2 p}\right) d s \\
& \leq C\|t-k \delta\|^{p} .
\end{aligned}
$$

Next, by Kunita's inequality [19, Theorem 4.4.23], we have

$$
\begin{aligned}
\mathbb{E} I_{4} & \leq C \mathbb{E} \int_{k \delta}^{t} \int_{\mathbb{Z}}\left\|S_{t-s} h\left(X_{s}^{\epsilon}, z\right)\right\|^{2 p} v(d z) d s+C \mathbb{E}\left\{\int_{k \delta}^{t} \int_{\mathbb{Z}}\left\|S_{t-s} h\left(X_{s}^{\epsilon}, z\right)\right\|^{2} v(d z) d s\right\}^{p} \\
& \leq C \mathbb{E} \int_{k \delta}^{t}\left(1+\left\|X_{s}^{\epsilon}\right\|^{2 p}\right) d s \\
& \leq C\|t-k \delta\| .
\end{aligned}
$$

Finally, we will estimate the first term $I_{1}$ of (3.5). To proceed, we define three functions and establish a key lemma.

Define

$$
\begin{aligned}
& \Upsilon_{t}^{\epsilon}:=\int_{0}^{t} S_{t-s} f\left(X_{s}^{\epsilon}, Y_{s}^{\epsilon}\right) d s, \\
& \Phi_{t}^{\epsilon}:=\int_{0}^{t} S_{t-s} g\left(X_{s}^{\epsilon}\right) d W_{s}^{1}, \\
& \Psi_{t}^{\epsilon}:=\int_{0}^{t} \int_{\mathbb{Z}} S_{t-s} h\left(X_{s-}^{\epsilon}, z\right) \tilde{N}_{1}(d s, d z) .
\end{aligned}
$$

Since the semigroup $\left\{S_{t}\right\}_{t \geq 0}$ is analytic, the trajectories of $\Upsilon_{t}^{\epsilon}, \Phi_{t}^{\epsilon}$ and $\Psi_{t}^{\epsilon}$ are Hölder continuous-valued. We will give some estimations of the slow component $X_{t}^{\epsilon}$ as a value process in $D\left((-\mathbb{A})^{\alpha}\right), \alpha \in\left(0, \frac{1}{8}\right)$.

Remark 3.4 In this paper, we assume that $\frac{1}{1-4 \alpha}<p<\frac{1}{4 \alpha}$ and $\alpha \in\left(0, \frac{1}{8}\right)$.

Lemma 3.5 For any $t \in[0, T]$ and $\frac{1}{1-4 \alpha}<p<\frac{1}{4 \alpha}, \alpha \in\left(0, \frac{1}{8}\right)$, there exists a constant $C_{\alpha, p, T}$ such that

$$
\mathbb{E}\left\|X_{t}^{\epsilon}\right\|_{\alpha}^{2 p} \leq C_{\alpha, p, T}
$$

Proof The estimations of $\Upsilon_{t}^{\epsilon}, \Phi_{t}^{\epsilon}$ can be obtained from [17]. Here, we give the proof of the third term $\Psi_{t}^{\epsilon}$. For the third term, by the factorization formula, we have

$$
\Psi_{t}^{\epsilon}=C_{\alpha} \int_{0}^{t}(t-s)^{\alpha-1} S_{t-s} U_{\alpha}^{\epsilon}(s) d s,
$$

with $U_{\alpha}^{\epsilon}(s)=\int_{0}^{s} \int_{\mathbb{Z}}(s-r)^{-\alpha} S_{s-r} h\left(X_{r-}^{\epsilon}, z\right) \tilde{N}_{1}(d r, d z)$. 
Note that, for any $\frac{1}{1-4 \alpha}<p<\frac{1}{4 \alpha}, \alpha \in\left(0, \frac{1}{8}\right)$, we have

$$
\begin{aligned}
\left\|\Psi_{t}^{\epsilon}\right\|_{\alpha}^{2 p} & \leq C_{\alpha}\left[\int_{0}^{t}(t-s)^{\alpha-1}\left\|U_{\alpha}^{\epsilon}(s)\right\|_{\alpha} d s\right]^{2 p} \\
& \leq C_{\alpha} \sup _{0 \leq s \leq t}\left\|U_{\alpha}^{\epsilon}(s)\right\|_{\alpha}^{2 p}\left[\int_{0}^{t}(t-s)^{\alpha-1} d s\right]^{2 p} \\
& \leq C_{\alpha, p, T} \sup _{0 \leq s \leq t}\left\|U_{\alpha}^{\epsilon}(s)\right\|_{\alpha}^{2 p} .
\end{aligned}
$$

Next, for any $t>0$, the operator $(-\mathbb{A})^{\alpha} S_{t}$ is bounded and its operator norm $\left\|(-\mathbb{A})^{\alpha} S_{t}\right\| \leq$ $M_{\alpha} t^{-\alpha}$ [48]. Then, by Kunita's first inequality [19, Theorem 4.4.23], Hölder's inequality and Lemma 3.2, we have

$$
\begin{aligned}
\mathbb{E}\left\|\Psi_{t}^{\epsilon}\right\|_{\alpha}^{2 p} \leq & C_{\alpha, p, T} \mathbb{E} \sup _{0 \leq s \leq t}\left\|\int_{0}^{s} \int_{\mathbb{Z}}(s-r)^{-\alpha}(-\mathbb{A})^{\alpha} S_{s-r} h\left(X_{r-}^{\epsilon}, z\right) \tilde{N}_{1}(d r, d z)\right\|^{2 p} \\
\leq & C_{\alpha, p, T} \mathbb{E} \int_{0}^{t} \int_{\mathbb{Z}}(s-r)^{-4 p \alpha}\left\|h\left(X_{r}^{\epsilon}, z\right)\right\|^{2 p} v(d z) d r \\
& +C_{\alpha, p, T} \mathbb{E}\left[\int_{0}^{t} \int_{\mathbb{Z}}(s-r)^{-4 \alpha}\left\|h\left(X_{r}^{\epsilon}, z\right)\right\|^{2} v(d z) d r\right]^{p} \\
\leq & C_{\alpha, p, T} \mathbb{E}\left[\sup _{0 \leq r \leq t} \int_{\mathbb{Z}}\left\|h\left(X_{r}^{\epsilon}, z\right)\right\|^{2 p} v(d z)\right] \int_{0}^{t} \int_{\mathbb{Z}}(s-r)^{-4 p \alpha} d r \\
& +C_{\alpha, p, T}\left[\int_{0}^{t}(s-r)^{\frac{4 p \alpha}{1-p}} d r\right]^{p-1} \mathbb{E} \int_{0}^{t}\left[\int_{\mathbb{Z}}\left\|h\left(X_{r}^{\epsilon}, z\right)\right\|^{2} v(d z)\right]^{p} d r \\
\leq & C_{\alpha, p, T} \int_{0}^{T} \mathbb{E}\left[1+\left\|X_{r}^{\epsilon}\right\|^{2 p}\right] d r \\
\leq & C_{\alpha, p, T} .
\end{aligned}
$$

Then, by $\left\|S_{t} X_{0}\right\|_{\alpha}^{2 p} \leq\left\|X_{0}\right\|_{\alpha}^{2 p}$, we have

$$
\mathbb{E}\left\|X_{t}^{\epsilon}\right\|_{\alpha}^{2 p} \leq C_{\alpha, p, T}
$$

This is the proof of Lemma 3.5.

To proceed, we give the estimation of $I_{1}$. According to [48], there exists a constant $C_{\alpha}>0$ such that for all $x \in D\left((-\mathbb{A})^{\alpha}\right)$,

$$
I_{1}=\left\|X_{k \delta}^{\epsilon}\left(S_{t-k \delta}-\mathbb{I}\right)\right\| \leq C_{\alpha}\|t-k \delta\|^{\alpha}\left\|X_{k \delta}^{\epsilon}\right\|_{\alpha}
$$

and then, according to Lemma 3.5, we deduce

$$
\begin{aligned}
\mathbb{E} I_{1} & =4^{2 p-1}\left\|X_{k \delta}^{\epsilon}\left(S_{t-k \delta}-\mathbb{I}\right)\right\|^{2 p} \\
& \leq 4^{2 p-1} C_{\alpha}\|t-k \delta\|^{2 p \alpha} \mathbb{E}\left\|X_{k \delta}^{\epsilon}\right\|_{\alpha}^{2 p} \\
& \leq C_{\alpha, p, T}\|t-k \delta\|^{2 p \alpha} .
\end{aligned}
$$


It then follows from (3.6)-(3.9) that

$$
\begin{aligned}
\mathbb{E}\left\|X_{t}^{\epsilon}-X_{k \delta}^{\epsilon}\right\|^{2 p} & \leq C_{\alpha, p, T}\|t-k \delta\|^{2 p \alpha}+C_{\alpha, p, T}\|t-k \delta\| \\
& \leq C_{\alpha, p, T}\|t-k \delta\|^{2 p \alpha} \\
& \leq C_{\alpha, p, T} \delta^{2 p \alpha} .
\end{aligned}
$$

Note that the result also holds for $p=1$ [45]:

$$
\mathbb{E}\left\|X_{t}^{\epsilon}-X_{k \delta}^{\epsilon}\right\|^{2} \leq C_{\alpha, p, T} \delta^{2 \alpha}
$$

Next, from the definitions of $Y_{t}^{\epsilon},(2.3)$ and $\hat{Y}_{t}^{\epsilon}$ (3.3), by energy identities (2.6), for $t \in$ $[k \delta, \min \{(k+1) \delta, T\}], \mathbb{E}\left\|Y_{t}^{\epsilon}-\hat{Y}_{t}^{\epsilon}\right\|^{2 p}$ will be estimated:

$$
\begin{aligned}
\mathbb{E} \| Y_{t}^{\epsilon}- & \hat{Y}_{t}^{\epsilon} \|^{2 p} \\
= & \frac{2 p}{\epsilon} \mathbb{E} \int_{k \delta}^{t}\left\|Y_{s}^{\epsilon}-\hat{Y}_{s}^{\epsilon}\right\|^{2 p-2}\left\langle\mathbb{A} Y_{s}^{\epsilon}-\mathbb{A} \hat{Y}_{s}^{\epsilon}, Y_{s}^{\epsilon}-\hat{Y}_{s}^{\epsilon}\right\rangle d s \\
& +\frac{2 p}{\epsilon} \mathbb{E} \int_{k \delta}^{t}\left\|Y_{s}^{\epsilon}-\hat{Y}_{s}^{\epsilon}\right\|^{2 p-2}\left\langle F\left(X_{s}^{\epsilon}, Y_{s}^{\epsilon}\right)-F\left(X_{k \delta}^{\epsilon}, \hat{Y}_{s}^{\epsilon}\right), Y_{s}^{\epsilon}-\hat{Y}_{s}^{\epsilon}\right\rangle_{\mathbb{H}} d s \\
& +\frac{2 p(p-1)}{\epsilon} \mathbb{E} \int_{k \delta}^{t}\left\|Y_{s}^{\epsilon}-\hat{Y}_{s}^{\epsilon}\right\|^{2 p-2}\left\|G\left(X_{s}^{\epsilon}, \hat{Y}_{s}^{\epsilon}\right)-G\left(X_{k \delta}^{\epsilon}, Y_{s}^{\epsilon}\right)\right\|^{2} d s \\
& +\frac{1}{\epsilon} \mathbb{E} \int_{k \delta}^{t} \int_{\mathbb{Z}}\left[\left\|\left(Y_{s}^{\epsilon}-\hat{Y}_{s}^{\epsilon}\right)+\left(H\left(X_{s}^{\epsilon}, Y_{s}^{\epsilon}, z\right)-H\left(X_{k \delta}^{\epsilon}, \hat{Y}_{s}^{\epsilon}, z\right)\right)\right\|^{2 p}\right. \\
& \left.-\left\|Y_{s}^{\epsilon}-\hat{Y}_{s}^{\epsilon}\right\|^{2 p}\right] v(d z) d s \\
& -\frac{2 p}{\epsilon} \mathbb{E} \int_{k \delta}^{t} \int_{\mathbb{Z}}\left\|Y_{s}^{\epsilon}-\hat{Y}_{s}^{\epsilon}\right\|^{2 p-2}\left\langle H\left(X_{s}^{\epsilon}, Y_{s}^{\epsilon}, z\right)\right. \\
& \left.-H\left(X_{k \delta}^{\epsilon}, \hat{Y}_{s}^{\epsilon}, z\right), Y_{s}^{\epsilon}-\hat{Y}_{s}^{\epsilon}\right\rangle_{\mathbb{H}} v(d z) d s \\
= & J_{1}+J_{2}+J_{3}+J_{4}+J_{5} .
\end{aligned}
$$

First of all, from Assumptions 1-3 and Young's inequality, it is easy to get

$$
\begin{aligned}
J_{1}+J_{2}+J_{3} & \leq \frac{C}{\epsilon} \mathbb{E} \int_{k \delta}^{t}\left(\left\|X_{s}^{\epsilon}-X_{k \delta}^{\epsilon}\right\|^{2 p}+\left\|Y_{s}^{\epsilon}-\hat{Y}_{s}^{\epsilon}\right\|^{2 p}\right) d s \\
& \leq \frac{C}{\epsilon} \mathbb{E} \int_{k \delta}^{t}\left\|Y_{s}^{\epsilon}-\hat{Y}_{s}^{\epsilon}\right\|^{2 p} d s+\frac{C_{\alpha, p, T} \delta^{2 p \alpha+1}}{\epsilon} .
\end{aligned}
$$

Then, by equality (3.1), we have

$$
\begin{aligned}
J_{4}+J_{5} & =\sum_{i=2}^{2 p} C_{i}^{2 p} \mathbb{E} \int_{k \delta}^{t} \int_{\mathbb{Z}}\left\|Y_{s}^{\epsilon}-\hat{Y}_{s}^{\epsilon}\right\|^{2 p-i}\left\|H\left(X_{s}^{\epsilon}, Y_{s}^{\epsilon}, z\right)-H\left(X_{k \delta}^{\epsilon}, \hat{Y}_{s}^{\epsilon}, z\right)\right\|^{i} v(d z) d s \\
& \leq \frac{C}{\epsilon} \sum_{i=2}^{2 p} \mathbb{E} \int_{k \delta}^{t}\left\|Y_{s}^{\epsilon}-\hat{Y}_{s}^{\epsilon}\right\|^{2 p-i}\left(\left\|X_{s}^{\epsilon}-X_{k \delta}^{\epsilon}\right\|^{i}+\left\|Y_{s}^{\epsilon}-\hat{Y}_{s}^{\epsilon}\right\|^{i}\right) d s \\
& \leq \frac{C}{\epsilon} \mathbb{E} \int_{k \delta}^{t}\left\|Y_{s}^{\epsilon}-\hat{Y}_{s}^{\epsilon}\right\|^{2 p} d s+\frac{C_{\alpha, p, T} \delta^{2 p \alpha+1}}{\epsilon} .
\end{aligned}
$$


Therefore, for $t \in[k \delta, \min \{(k+1) \delta, T\}]$, we obtain

$$
\begin{aligned}
\mathbb{E}\left\|Y_{t}^{\epsilon}-\hat{Y}_{t}^{\epsilon}\right\|^{2 p} & \leq \frac{C}{\epsilon} \mathbb{E} \int_{k \delta}^{t}\left\|Y_{s}^{\epsilon}-\hat{Y}_{s}^{\epsilon}\right\|^{2 p} d s+\frac{C_{\alpha, p, T} \delta^{2 p \alpha+1}}{\epsilon} \\
& \leq \frac{C_{\alpha, p, T} \delta^{2 p \alpha+1}}{\epsilon} e^{\frac{C}{\epsilon} \delta} .
\end{aligned}
$$

To proceed, we give another key lemma to complete the proof of Step 1.

Lemma 3.6 For any $t \in[0, T]$, and $\frac{1}{1-4 \alpha}<p<\frac{1}{4 \alpha}, \alpha \in\left(0, \frac{1}{8}\right)$, we have

$$
\mathbb{E} \sup _{0 \leq t \leq T}\left\|X_{t}^{\epsilon}-\hat{X}_{t}^{\epsilon}\right\|^{2 p} \leq C_{\alpha, p, T}\left(\frac{\delta^{2 p \alpha+1}}{\epsilon} e^{\frac{C}{\epsilon} \delta}+\delta^{2 p \alpha}\right) e^{C_{p} T}
$$

Proof We begin with

$$
\begin{aligned}
\left\|X_{t}^{\epsilon}-\hat{X}_{t}^{\epsilon}\right\|^{2 p}= & 2 p \int_{0}^{t}\left\|X_{s}^{\epsilon}-\hat{X}_{s}^{\epsilon}\right\|^{2 p-2}\left\langle\mathbb{A} X_{s}^{\epsilon}-\mathbb{A} \hat{X}_{s}^{\epsilon}, X_{s}^{\epsilon}-\hat{X}_{s}^{\epsilon}\right\rangle d s \\
& +2 p \int_{0}^{t}\left\|X_{s}^{\epsilon}-\hat{X}_{s}^{\epsilon}\right\|^{2 p-2}\left\langle f\left(X_{s}^{\epsilon}, Y_{s}^{\epsilon}\right)-f\left(X_{[s / \delta] \delta}^{\epsilon}, \hat{Y}_{s}^{\epsilon}\right), X_{s}^{\epsilon}-\hat{X}_{s}^{\epsilon}\right\rangle_{\mathbb{H}} d s
\end{aligned}
$$

Thanks to Lemma 3.6 and (3.11), for any $u \in[0, T]$, we get

$$
\begin{aligned}
\mathbb{E} \sup _{0 \leq t \leq u}\left\|X_{t}^{\epsilon}-\hat{X}_{t}^{\epsilon}\right\|^{2 p} \leq & C_{p} \int_{0}^{t} \mathbb{E} \sup _{0 \leq r \leq s}\left\|X_{r}^{\epsilon}-\hat{X}_{r}^{\epsilon}\right\|^{2 p} d s \\
& +C_{p} \int_{0}^{u} \mathbb{E} \sup _{0 \leq r \leq s}\left\|X_{r}^{\epsilon}-X_{[r / \delta] \delta}^{\epsilon}\right\|^{2 p} d s \\
& +C_{p} \int_{0}^{t} \mathbb{E}\left\|Y_{s}^{\epsilon}-\hat{Y}_{s}^{\epsilon}\right\|^{2 p} d s \\
\leq & C_{p} \int_{0}^{u} \mathbb{E} \sup _{0 \leq r \leq u}\left\|X_{r}^{\epsilon}-\hat{X}_{r}^{\epsilon}\right\|^{2 p} d s+C_{\alpha, p, T} \delta^{2 p \alpha} \\
& +\frac{C_{\alpha, p, T} \delta^{2 p \alpha+1}}{\epsilon} e^{\frac{C}{\epsilon} \delta} .
\end{aligned}
$$

With the help of Gronwall's inequality, we have

$$
\mathbb{E} \sup _{0 \leq t \leq T}\left\|X_{t}^{\epsilon}-\hat{X}_{t}^{\epsilon}\right\|^{2 p} \leq C_{\alpha, p, T}\left(\frac{\delta^{2 p \alpha+1}}{\epsilon} e^{\frac{C}{\epsilon} \delta}+\delta^{2 p \alpha}\right) e^{C_{p} T}
$$

This is the proof of Lemma 3.6.

Step 2. In this step, we will estimate $\mathbb{E} \sup _{0 \leq t \leq T}\left\|\hat{X}_{t}^{\epsilon}-\bar{X}_{t}\right\|^{2 p}$. It follows from the definitions of $\bar{X}_{t}$ and $\hat{X}_{t}^{\epsilon}$ that

$$
\begin{aligned}
\hat{X}_{t}^{\epsilon}-\bar{X}_{t}= & \int_{0}^{t} S_{t-s}\left[f\left(X_{k \delta}^{\epsilon}, \hat{Y}_{s}^{\epsilon}\right)-\bar{f}\left(X_{s}^{\epsilon}\right)\right] d s \\
& +\int_{0}^{t} S_{t-s}\left[\bar{f}\left(X_{s}^{\epsilon}\right)-\bar{f}\left(\hat{X}_{s}^{\epsilon}\right)\right] d s+\int_{0}^{t} S_{t-s}\left[\bar{f}\left(\hat{X}_{s}^{\epsilon}\right)-\bar{f}\left(\bar{X}_{s}\right)\right] d s
\end{aligned}
$$




$$
\begin{aligned}
& +\int_{0}^{t} S_{t-s}\left[g\left(X_{s}^{\epsilon}\right)-g\left(\hat{X}_{s}^{\epsilon}\right)\right] d W_{s}^{1}+\int_{0}^{t} S_{t-s}\left[g\left(\hat{X}_{s}^{\epsilon}\right)-g\left(\bar{X}_{s}\right)\right] d W_{s}^{1} \\
& +\int_{0}^{t} \int_{\mathbb{Z}} S_{t-s}\left[h\left(X_{s-}^{\epsilon}, z\right)-h\left(\hat{X}_{s-}^{\epsilon}, z\right)\right] \tilde{N}_{1}(d s, d z) \\
& +\int_{0}^{t} \int_{\mathbb{Z}} S_{t-s}\left[h\left(\hat{X}_{s-}^{\epsilon}, z\right)-h\left(\bar{X}_{s-}, z\right)\right] \tilde{N}_{1}(d s, d z) \\
& =\sum_{i=1}^{7} \Xi_{i}(t) .
\end{aligned}
$$

Using Hölder's inequality, the contractive property of semigroup $S_{t}$, and the globally Lipschitz continuity of $\bar{f}$, for any $u \in[0, T]$, we obtain

$$
\begin{aligned}
\mathbb{E} \sum_{i=2,4} \sup _{0 \leq t \leq u}\left\|\Xi_{i}(t)\right\|^{2 p} & \leq C_{T} \int_{0}^{u} \mathbb{E} \sup _{0 \leq r \leq s}\left\|X_{r}^{\epsilon}-\hat{X}_{r}^{\epsilon}\right\|^{2 p} d s \\
& \leq C_{\alpha, p, T}\left(\frac{\delta^{2 p \alpha+1}}{\epsilon} e^{\frac{C}{\epsilon} \delta}+\delta^{2 p \alpha}\right) e^{C_{p} T}
\end{aligned}
$$

Similarly, it is also easy to derive that the estimate for any $u \in[0, T]$,

$$
\mathbb{E} \sum_{i=3,5} \sup _{0 \leq t \leq u}\left\|\Xi_{i}(t)\right\|^{2 p} \leq C_{T} \int_{0}^{u} \mathbb{E} \sup _{0 \leq r \leq s}\left\|\hat{X}_{r}^{\epsilon}-\bar{X}_{r}\right\|^{2 p} d s
$$

Now, by Kunita's first inequality [19, Theorem 4.4.23] and Hölder's inequality, we have

$$
\begin{aligned}
\mathbb{E} \sup _{0 \leq t \leq u}\left\|\Xi_{6}(t)\right\|^{2 p}= & \mathbb{E} \sup _{0 \leq t \leq u}\left[\int_{0}^{t} \int_{\mathbb{Z}} S_{t-s}\left[h\left(X_{s-}^{\epsilon}, z\right)-h\left(\hat{X}_{s-}^{\epsilon}, z\right)\right] \tilde{N}_{1}(d s, d z)\right]^{2 p} \\
\leq & C_{p} \mathbb{E} \int_{0}^{u} \int_{\mathbb{Z}}\left\|S_{t-s}\left[h\left(X_{s}^{\epsilon}, z\right)-h\left(\hat{X}_{s}^{\epsilon}, z\right)\right]\right\|^{2 p} v(d z) d t \\
& +C_{p} \mathbb{E}\left[\int_{0}^{u} \int_{\mathbb{Z}}\left\|S_{t-s}\left[h\left(X_{s}^{\epsilon}, z\right)-h\left(\hat{X}_{s}^{\epsilon}, z\right)\right]\right\|^{2} v(d z) d t\right]^{p} \\
\leq & C_{p, T} \int_{0}^{u} \mathbb{E} \sup _{0 \leq r \leq s}\left\|\hat{X}_{r}^{\epsilon}-X_{r}^{\varepsilon}\right\|^{2 p} d s \\
\leq & C_{\alpha, p, T}\left(\frac{\delta^{2 p \alpha+1}}{\epsilon} e^{\frac{C}{\epsilon} \delta}+\delta^{2 p \alpha}\right) e^{C_{p} T}
\end{aligned}
$$

and

$$
\begin{aligned}
\mathbb{E} \sup _{0 \leq t \leq u}\left\|\Xi_{6}(t)\right\|^{2 p}= & \mathbb{E} \sup _{0 \leq t \leq u}\left[\int_{0}^{t} \int_{\mathbb{Z}} S_{t-s}\left[h\left(\bar{X}_{s-}, z\right)-h\left(\hat{X}_{s-}^{\epsilon}, z\right)\right] \tilde{N}_{1}(d s, d z)\right]^{2 p} \\
\leq & C_{p} \mathbb{E} \int_{0}^{u} \int_{\mathbb{Z}}\left\|S_{t-s}\left[h\left(\bar{X}_{s-}, z\right)-h\left(\hat{X}_{s-}^{\epsilon}, z\right)\right]\right\|^{2 p} v(d z) d t \\
& +C_{p} \mathbb{E}\left[\int_{0}^{u} \int_{\mathbb{Z}}\left\|S_{t-s}\left[h\left(\bar{X}_{s-}, z\right)-h\left(\hat{X}_{s-}^{\epsilon}, z\right)\right]\right\|^{2} v(d z) d t\right]^{p} \\
\leq & C_{p, T} \int_{0}^{u} \mathbb{E} \sup _{0 \leq r \leq s}\left\|\hat{X}_{r}^{\epsilon}-\bar{X}_{r}\right\|^{2 p} d s .
\end{aligned}
$$


Next, to deal with the first term, by the boundedness of the functions $f, \bar{f}$, we have

$$
\begin{aligned}
\mathbb{E} \sup _{0 \leq t \leq T}\left\|\Xi_{1}(t)\right\|^{2 p} & \leq \mathbb{E} \sup _{0 \leq t \leq T}\left\|\int_{0}^{t} S_{t-s}\left[f\left(X_{[s / \delta] \delta}^{\epsilon}, \hat{Y}_{s}^{\epsilon}\right)-\bar{f}\left(X_{s}^{\epsilon}\right)\right] d s\right\|^{2 p} \\
& \leq C_{T} \mathbb{E} \sup _{0 \leq t \leq T}\left\|\int_{0}^{t} S_{t-s}\left[f\left(X_{[s / \delta] \delta}^{\epsilon}, \hat{Y}_{s}^{\epsilon}\right)-\bar{f}\left(X_{s}^{\epsilon}\right)\right] d s\right\|^{2} .
\end{aligned}
$$

For $t \in[k \delta, \min \{(k+1) \delta, T\}]$, we write

$$
\begin{aligned}
\Xi_{1}(t)= & \sum_{k=0}^{k-1} \int_{k \delta}^{(k+1) \delta} S_{t-s}\left[f\left(X_{k \delta}^{\epsilon}, \hat{Y}_{s}^{\epsilon}\right)-\bar{f}\left(X_{k \delta}^{\epsilon}\right)\right] d s \\
& +\sum_{k=0}^{k-1} \int_{k \delta}^{(k+1) \delta} S_{t-s}\left[\bar{f}\left(X_{k \delta}^{\epsilon}\right)-\bar{f}\left(X_{s}^{\epsilon}\right)\right] d s \\
& +\int_{k \delta}^{t} S_{t-s}\left[f\left(X_{k \delta}^{\epsilon}, \hat{Y}_{s}^{\epsilon}\right)-\bar{f}\left(X_{s}^{\epsilon}\right)\right] d s \\
= & \sum_{i=1}^{3} \Xi_{1 i}(t) .
\end{aligned}
$$

From (3.10), it follows that

$$
\begin{aligned}
\mathbb{E} \sup _{0 \leq t \leq u}\left\|\Xi_{12}(t)\right\|^{2} & =\mathbb{E} \sup _{0 \leq t \leq u}\left\|\sum_{k=0}^{k-1} \int_{k \delta}^{(k+1) \delta} S_{t-s}\left[\bar{f}\left(X_{k \delta}^{\epsilon}\right)-\bar{f}\left(X_{s}^{\epsilon}\right)\right] d s\right\|^{2} \\
& \leq C_{T} \int_{0}^{T} \mathbb{E}\left\|X_{s}^{\epsilon}-X_{k \delta}^{\epsilon}\right\|^{2} d s \\
& \leq C_{\alpha, T} \delta^{2 \alpha}
\end{aligned}
$$

and by Assumption 3, we have

$$
\begin{aligned}
& \mathbb{E} \sup _{0 \leq t \leq u}\left\|\Xi_{13}(t)\right\|^{2}=\mathbb{E} \sup _{0 \leq t \leq u}\left\|\int_{k \delta}^{t} S_{t-s}\left[f\left(X_{k \delta}^{\epsilon}, \hat{Y}_{s}^{\epsilon}\right)-\bar{f}\left(X_{s}^{\epsilon}\right)\right] d s\right\|^{2} \\
& \leq C_{T} \delta \text {. }
\end{aligned}
$$

Lemma 3.7 Suppose that Assumptions 1-3 hold. Then there is a constant $C>0$ such that we have

$$
\begin{aligned}
\mathbb{E} \sup _{0 \leq t \leq T}\left\|\Xi_{11}(t)\right\|^{2} & =\mathbb{E} \sup _{0 \leq t \leq T}\left\|\sum_{k=0}^{k-1} \int_{k \delta}^{(k+1) \delta} S_{t-s}\left[f\left(X_{k \delta}^{\epsilon}, \hat{Y}_{s}^{\epsilon}\right)-\bar{f}\left(X_{k \delta}^{\epsilon}\right)\right] d s\right\|^{2} \\
& \leq C{ }_{\frac{\delta}{\epsilon}}^{\delta}
\end{aligned}
$$

where $C$ is independent of $(\delta, \epsilon)$.

Proof See Appendix B. 
Now, using the above estimation (3.16)-(3.18) and Lemma 3.7, we obtain

$$
\begin{aligned}
\mathbb{E} \sup _{0 \leq t \leq u}\left\|\Xi_{1}(t)\right\|^{2 p} & \leq C \delta+C_{\alpha, T} \delta^{2 \alpha}+C_{T} \frac{\epsilon}{\delta} \\
& \leq C_{\alpha, T} \delta^{2 \alpha}+C_{T} \frac{\epsilon}{\delta}
\end{aligned}
$$

As regards the above discussion, from (3.12), (3.13) and (3.19), through Gronwall's inequality, it is easy to see that

$$
\begin{aligned}
\mathbb{E} \sup _{0 \leq t \leq u}\left\|\hat{X}_{t}^{\epsilon}-\bar{X}_{t}\right\|^{2 p} \leq & C_{\alpha, T} \delta^{2 \alpha}+C_{T} \frac{\epsilon}{\delta}+C_{\alpha, p, T}\left(\frac{\delta^{2 p \alpha+1}}{\epsilon} e^{\frac{C}{\epsilon} \delta}+\delta^{2 p \alpha}\right) e^{C_{p} T} \\
& +C_{T} \int_{0}^{u} \mathbb{E} \sup _{0 \leq r \leq s}\left\|\hat{X}_{r}^{\epsilon}-\bar{X}_{r}\right\|^{2 p} d r
\end{aligned}
$$

Therefore, by Gronwall's inequality, we have

$$
\mathbb{E} \sup _{0 \leq t \leq u}\left\|\hat{X}_{t}^{\epsilon}-\bar{X}_{t}\right\|^{2 p} \leq\left[C_{\alpha, T} \delta^{2 \alpha}+C_{T} \frac{\epsilon}{\delta}+C_{\alpha, p, T}\left(\frac{\delta^{2 p \alpha+1}}{\epsilon} e^{\frac{C}{\epsilon} \delta}+\delta^{2 p \alpha}\right) e^{C_{p} T}\right] e^{C_{p, T}} .
$$

Step 3. According to Step 1 and Step 2, we have

$$
\begin{aligned}
\mathbb{E} \sup _{0 \leq t \leq T}\left\|X_{t}^{\varepsilon}-\bar{X}_{t}\right\|^{2 p}= & \mathbb{E} \sup _{0 \leq t \leq T}\left\|X_{t}^{\varepsilon}-\hat{X}_{t}^{\varepsilon}+\hat{X}_{t}^{\varepsilon}-\bar{X}_{t}\right\|^{2 p} \\
\leq & 2^{2 p-1} \mathbb{E} \sup _{0 \leq t \leq T}\left\|X_{t}^{\varepsilon}-\hat{X}_{t}^{\varepsilon}\right\|^{2 p}+2^{2 p-1} \mathbb{E} \sup _{0 \leq t \leq T}\left\|\hat{X}_{t}^{\varepsilon}-\bar{X}_{t}\right\|^{2 p} \\
\leq & {\left[C_{\alpha, T} \delta^{2 \alpha}+C_{T} \frac{\epsilon}{\delta}+C_{\alpha, p, T}\left(\frac{\delta^{2 p \alpha+1}}{\epsilon} e^{\frac{C}{\epsilon} \delta}+\delta^{2 p \alpha}\right) e^{C_{p} T}\right] e^{C_{p, T}} } \\
& +C_{\alpha, p, T}\left(\frac{\delta^{2 p \alpha+1}}{\epsilon} e^{\frac{C}{\epsilon} \delta}+\delta^{2 p \alpha}\right) e^{C_{p} T} \\
\leq & C_{\alpha, p, T}\left[\delta^{2 \alpha}+\delta^{2 p \alpha}+\frac{\epsilon}{\delta}+\frac{\delta^{2 p \alpha+1}}{\epsilon} e^{\frac{C}{\epsilon} \delta}\right]
\end{aligned}
$$

Thus, for $t \in[0, T]$, selecting $\delta=\epsilon \sqrt{-\ln \epsilon}$, we obtain

$$
\mathbb{E} \sup _{0 \leq t \leq T}\left\|\hat{X}_{t}^{\epsilon}-\bar{X}_{t}\right\|^{2 p} \rightarrow 0
$$

as $\epsilon \rightarrow 0, t \in[0, T]$.

This is the proof of Theorem 3.6.

Remark 3.8 To compare with the work of $\mathrm{Xu}$ and Miao [45] that the $\mathbb{L}^{2}$-strong averaging principle for slow-fast SPDEs with Poisson random measures was established, in this paper, we cope with high order moments which possess a good robustness and can be applied in computations in statistics, finance and other aspects.

\section{Appendix A}

In this appendix, we shall show the ergodicity of the fast equation with frozen slow component for the reader's convenience [46]. For a fixed $x \in \mathbb{H}$, consider the problem associated 
with fast motion with frozen show component

$$
d Y_{t}=\left[\mathbb{A} Y_{t}+F\left(x, Y_{t}\right)\right] d t+G\left(x, Y_{t}\right) d \bar{W}_{t}+\int_{\mathbb{Z}} H\left(x, Y_{t-}, z\right) \bar{N}(d t, d z), \quad Y_{0}=y,
$$

where $\bar{W}_{t}$ is a Wiener process, $\bar{N}(d t, d z)$ is a Poisson random measure with the compensator $v(d z) d t$, and they are defined on the stochastic basis $(\Omega, \mathcal{F}, \mathbb{P})$. Then, for any fixed $x \in \mathbb{H}$ and any $y \in \mathbb{H}$, (A.1) has a unique mild solution which will be denoted by $Y_{t}^{x, y}$.

Appendix A.1 ([46]) We assume that Assumptions 1-3 hold. Then there exists a constant C such that

$$
\left\|\mathbb{E} f\left(x, Y_{t}^{x, y}\right)-\bar{f}(x)\right\|^{2} \leq C e^{-\eta t}\left(1+\|x\|^{2}+\|y\|^{2}\right),
$$

where $\eta=\alpha_{1}-C_{F}-C_{G}-C_{H}>0, \bar{f}(x)$ also satisfies the globally Lipschitz condition (Assumption 1) and

$$
\bar{f}(x)=\int_{\mathbb{H}} f(x, y) \mu^{x}(d y), \quad x \in \mathbb{H}
$$

where $\mu^{x}$ denotes the unique invariant measure of (A.1).

Proof By the energy equality [45], we get

$$
\begin{aligned}
\frac{d}{d t} \mathbb{E}\left\|Y_{t}^{x, y}\right\|^{2} \leq & 2 \mathbb{E}\left\langle\mathbb{A} Y_{t}^{x, y}, Y_{t}^{x, y}\right\rangle+2 \mathbb{E}\left\langle F\left(x, Y_{t}^{x, y}\right)-F(x, 0), Y_{t}^{x, y}\right\rangle_{\mathbb{H}} \\
& +2 \mathbb{E}\left\langle F(x, 0), Y_{t}^{x, y}\right\rangle_{\mathbb{H}}+2 \mathbb{E}\left\|G\left(x, Y_{t}^{x, y}\right)-G(x, 0)\right\|^{2}+2 \mathbb{E}\|G(x, 0)\|^{2} \\
& +2 \mathbb{E} \int_{\mathbb{Z}}\left\|H\left(x, Y_{t}^{x, y}, z\right)-H(x, 0, z)\right\|^{2} v(d z) \\
& +2 \mathbb{E} \int_{\mathbb{Z}}\|H(x, 0, z)\|^{2} v(d z) \\
\leq & -2 \alpha_{1} \mathbb{E}\left\|Y_{t}^{x, y}\right\|^{2}+\left(C_{F}+1\right) \mathbb{E}\left\|Y_{t}^{x, y}\right\|^{2}+C \mathbb{E}\|F(x, 0)\|^{2} \\
& +\left(\alpha_{1}-1-C_{G}-C_{H}\right) \mathbb{E}\left\|Y_{t}^{x, y}\right\|^{2}+\left(2 C_{G}+2 C_{H}\right) \mathbb{E}\left\|Y_{t}^{x, y}\right\|^{2} \\
& +2 \mathbb{E}\|G(x, 0)\|^{2}+2 \mathbb{E} \int_{\mathbb{Z}}\|H(x, 0, z)\|^{2} v(d z) \\
\leq & -\eta \mathbb{E}\left\|Y_{t}^{x, y}\right\|^{2}+C\left(1+\|x\|^{2}\right),
\end{aligned}
$$

where $\eta=\alpha_{1}-C_{F}-C_{G}-C_{H}>0$.

Then by Assumption 1, Assumption 2, Remark 2.3, and the Gronwall inequality, we have

$$
\mathbb{E}\left\|Y_{t}^{x, y}\right\|^{2} \leq\|y\|^{2} e^{-\eta t}+C\left(1+\|x\|^{2}\right)
$$


Next, let $Y_{t}^{x, y^{\prime}}$ be a solution of (A.1) with the initial value $Y_{0}=y^{\prime}$. By the energy equality, we derive

$$
\begin{aligned}
\left\|Y_{t}^{x, y}-Y_{t}^{x, y^{\prime}}\right\|^{2}= & \left\|y-y^{\prime}\right\|^{2}+2 \int_{0}^{t}\left\langle\mathbb{A}\left(Y_{s}^{x, y}-Y_{s}^{x, y^{\prime}}\right), Y_{s}^{x, y}-Y_{s}^{x, y^{\prime}}\right\rangle d s \\
& +2 \int_{0}^{t}\left\langle F\left(x, Y_{s}^{x, y}\right)-F\left(x, Y_{s}^{x, y^{\prime}}\right), Y_{s}^{x, y}-Y_{s}^{x, y^{\prime}}\right\rangle_{\mathbb{H}} d s \\
& +2 \int_{0}^{t}\left\langle G\left(x, Y_{s}^{x, y}\right)-G\left(x, Y_{s}^{x, y^{\prime}}\right), Y_{s}^{x, y}-Y_{s}^{x, y^{\prime}}\right\rangle_{\mathbb{H}} d \bar{W}_{s} \\
& +\int_{0}^{t}\left\|G\left(x, Y_{s}^{x, y}\right)-G\left(x, Y_{s}^{x, y^{\prime}}\right)\right\|^{2} d s \\
& +\int_{0}^{t} \int_{\mathbb{Z}}\left\|H\left(x, Y_{s-}^{x, y}, z\right)-H\left(x, Y_{s-}^{x, y^{\prime}}, z\right)\right\|^{2} \bar{N}(d s, d z) \\
& +2 \int_{0}^{t} \int_{\mathbb{Z}}\left\langle H\left(x, Y_{s-}^{x, y}\right)-H\left(x, Y_{s-}^{x, y^{\prime}}\right), Y_{s-}^{x, y}-Y_{s-}^{x, y^{\prime}}\right\rangle_{\mathbb{H}} \bar{N}(d s, d z) \\
& +\int_{0}^{t} \int_{\mathbb{Z}}\left\|H\left(x, Y_{s}^{x, y}, z\right)-H\left(x, Y_{s}^{x, y^{\prime}}\right)\right\|^{2} v(d z) d s .
\end{aligned}
$$

With the aid of (2.2) and Assumptions 1 and 3, we have

$$
\mathbb{E}\left\|Y_{t}^{x, y}-Y_{t}^{x, y^{\prime}}\right\|^{2} \leq\left\|y-y^{\prime}\right\|^{2} e^{-\eta t}
$$

where $\eta=\alpha_{1}-C_{F}-C_{G}-C_{H}$.

Note that for any $x \in \mathbb{H}$ denoted by $P_{t}^{x}$ the Markov semigroup associated with (A.1) is defined by

$$
P_{t}^{x} \Psi\left(y^{\prime}\right)=\mathbb{E} \Psi\left(Y_{t}^{x, y^{\prime}}\right), \quad t \geq 0, y^{\prime} \in \mathbb{H}
$$

for any $\Psi \in \mathcal{B}_{b}(\mathbb{H})$ in the space of bounded functions on $\mathbb{H}$. We also recall a probability $\mu^{x}$ on $\mathbb{H}$, which is called an invariant measure for $\left(P_{t}^{x}\right)_{t \geq 0}$ if

$$
\int_{\mathbb{H}} P_{t}^{x} \Psi d \mu^{x}=\int_{\mathbb{H}} \Psi d \mu^{x}, \quad t \geq 0,
$$

for any bounded function $\Psi \in \mathcal{B}_{b}(\mathbb{H})$. As in $[49,50]$, it is possible to show the existence of the unique invariant measure $\mu^{x}$ for the semigroup $P_{t}^{x}$, which satisfies

$$
\int_{\mathbb{H}}\left\|y^{\prime}\right\|^{2} \mu^{x}\left(d y^{\prime}\right) \leq\left(1+\|x\|^{2}\right) .
$$

Furthermore, according to the Lipschitz assumption on $f$ and (A.3), we have

$$
\begin{aligned}
\left\|\mathbb{E} f\left(x, Y_{t}^{x, y}\right)-\int_{\mathbb{H}} f\left(x, y^{\prime}\right) \mu^{x}\left(d y^{\prime}\right)\right\|^{2} & =\left\|\int_{\mathbb{H}}\left[\mathbb{E} f\left(x, Y_{t}^{x, y}\right)-\mathbb{E} f\left(x, Y_{t}^{x, y^{\prime}}\right)\right] \mu^{x}\left(d y^{\prime}\right)\right\|^{2} \\
& \leq C \int_{\mathbb{H}} \mathbb{E}\left\|Y_{t}^{x, y}-Y_{t}^{x, y^{\prime}}\right\|^{2} \mu^{x}\left(d y^{\prime}\right) \\
& \leq C e^{-\frac{1}{2} \eta t} \int_{\mathbb{H}}\left\|y-y^{\prime}\right\|^{2} \mu^{x}\left(d y^{\prime}\right) \\
& \leq C e^{-\frac{1}{2} \eta t}\left(1+\|x\|^{2}+\|y\|^{2}\right) .
\end{aligned}
$$


This is the proof of Appendix A.1.

\section{Appendix B}

In this appendix, we show the proof of Lemma 3.7 for the reader's convenience.

First of all, we note that by a time shift transformation, it follows from the definition of $\hat{Y}_{s}^{\varepsilon}$ that for $s \in[0, \delta]$, the process $\hat{Y}_{k \delta+s}^{\varepsilon}$ coincides in distribution with the process $Y_{s / \varepsilon}^{X_{k \delta}^{\varepsilon}, Y_{k \delta}^{\varepsilon}}$ defined by (A.1) in Appendix A. We have

$$
\begin{aligned}
\hat{Y}_{s+k \delta}^{\varepsilon}= & Y_{k \delta}^{\varepsilon}+\frac{1}{\varepsilon} \int_{k \delta}^{k \delta+s} \mathbb{A} \hat{Y}_{u}^{\varepsilon} d u+\frac{1}{\varepsilon} \int_{k \delta}^{k \delta+s} F\left(X_{k \delta}^{\varepsilon}, \hat{Y}_{u}^{\varepsilon}\right) d u \\
& +\frac{1}{\sqrt{\varepsilon}} \int_{k \delta}^{k \delta+s} G\left(X_{k \delta}^{\varepsilon}, \hat{Y}_{u}^{\varepsilon}\right) d W_{u}^{2} \\
& +\int_{k \delta}^{k \delta+s} \int_{\mathbb{Z}} H\left(X_{k \delta}^{\varepsilon}, \hat{Y}_{u-}^{\varepsilon}, z\right) \tilde{N}_{2}^{\varepsilon}(d u, d z) \\
= & Y_{k \delta}^{\varepsilon}+\frac{1}{\varepsilon} \int_{0}^{s} \mathbb{A} \hat{Y}_{u+k \delta}^{\varepsilon} d u+\frac{1}{\varepsilon} \int_{0}^{s} F\left(X_{k \delta}^{\varepsilon}, \hat{Y}_{u+k \delta}^{\varepsilon}\right) d u \\
& +\frac{1}{\sqrt{\varepsilon}} \int_{0}^{s} G\left(X_{k \delta}^{\varepsilon}, \hat{Y}_{u+k \delta}^{\varepsilon}\right) d W_{u}^{2 *} \\
& +\int_{0}^{s} \int_{\mathbb{Z}} H\left(X_{k \delta}^{\varepsilon}, \hat{Y}_{u+k \delta}^{\varepsilon}, z\right) \tilde{N}_{2}^{\varepsilon, *}(d u, d z),
\end{aligned}
$$

where $W_{u}^{2 *}=W_{u+k \delta}^{2 *}-W_{k \delta}^{2}$ and $p_{u}^{2 *}=p_{u+k \delta}^{2}-p_{k \delta}^{2}$ are the shifts of $W_{u}^{2}$ and $p_{u}^{2}$, respectively. Let $\bar{W}_{u}$ be a Wiener process and independent of $W_{t}^{2}$ and $\bar{p}_{t}^{2}$ be a simple Poisson process and independent of $p_{t}^{1}$ and $p_{t}^{2}$. We construct a process $Y^{X_{k \delta}^{\varepsilon}, Y_{k \delta}^{\varepsilon}}$ by means of

$$
\begin{aligned}
& Y_{s / \varepsilon}^{X_{k \delta}^{\varepsilon}, Y_{k \delta}^{\varepsilon}}=Y_{k \delta}^{\varepsilon}+\int_{0}^{s / \varepsilon} \mathbb{A} Y_{u}^{X_{k \delta}^{\varepsilon}, Y_{k \delta}^{\varepsilon}} d u+\int_{0}^{s / \varepsilon} F\left(X_{k \delta}^{\varepsilon}, Y_{u}^{X_{k \delta}^{\varepsilon}, Y_{k \delta}^{\varepsilon}}\right) d u \\
& +\int_{0}^{s / \varepsilon} G\left(X_{k \delta}^{\varepsilon}, Y_{u}^{X_{k \delta}^{\varepsilon}, Y_{k \delta}^{\varepsilon}}\right) d \bar{W}_{u} \\
& +\int_{0}^{s / \varepsilon} \int_{\mathbb{Z}} H\left(X_{k \delta}^{\varepsilon}, Y_{u-}^{X_{k \delta}^{\varepsilon}, Y_{k \delta}^{\varepsilon}}, z\right) \bar{N}_{2}(d u, d z) \\
& =Y_{k \delta}^{\varepsilon}+\frac{1}{\varepsilon} \int_{0}^{s} \mathbb{A} Y_{u / \varepsilon}^{X_{k \delta}^{\varepsilon}, Y_{k \delta}^{\varepsilon}} d u+\frac{1}{\varepsilon} \int_{0}^{s} F\left(X_{k \delta}^{\varepsilon}, Y_{u / \varepsilon}^{X_{k \delta}^{\varepsilon}, Y_{k \delta}^{\varepsilon}}\right) d u \\
& +\frac{1}{\sqrt{\varepsilon}} \int_{0}^{s} G\left(X_{k \delta}^{\varepsilon}, Y_{u / \varepsilon}^{X_{k \delta}^{\varepsilon}, Y_{k \delta}^{\varepsilon}}\right) d \overline{\bar{W}}_{u} \\
& +\int_{0}^{s} \int_{\mathbb{Z}} H\left(X_{k \delta}^{\varepsilon}, Y_{u-\ell \varepsilon}^{X_{k \delta}^{\varepsilon}, Y_{k \delta}^{\varepsilon}}, z\right) \overline{\bar{N}}_{2}(d u, d z),
\end{aligned}
$$

where $\overline{\bar{W}}_{u}=\sqrt{\varepsilon} \bar{W}_{u / \varepsilon}$ and $\overline{\bar{p}}_{u}^{2}=\bar{p}_{u / \varepsilon}^{2}$ are the scaled versions of $\bar{W}_{u}$ and $\bar{p}_{u}^{2}$, respectively.

By comparison of (B.1) and (B.2), we have

$$
\left(X_{k \delta}^{\varepsilon}, \hat{Y}_{s+k \delta}^{\varepsilon}\right) \sim\left(X_{k \delta}^{\varepsilon}, Y_{s / \varepsilon}^{X_{k \delta}^{\varepsilon}, Y_{k \delta}^{\varepsilon}}\right), \quad s \in[0, \delta)
$$

where $\sim$ denotes a coincidence in the distribution sense. 
Prooffor Lemma 3.7 To proceed, as for $\Xi_{11}(t)$, by (B.3) we have

$$
\begin{aligned}
\mathbb{E} \sup _{0 \leq t \leq T}\left\|\Xi_{11}(t)\right\|^{2}= & \mathbb{E} \sup _{0 \leq t \leq T}\left\|\sum_{k=0}^{k-1} \int_{k \delta}^{(k+1) \delta} S_{t-s}\left[f\left(X_{k \delta}^{\epsilon}, \hat{Y}_{s}^{\epsilon}\right)-\bar{f}\left(X_{k \delta}^{\epsilon}\right)\right] d s\right\|^{2} \\
\leq & \lfloor T / \delta\rfloor^{2} \epsilon^{2} \max _{0 \leq k \leq\lfloor T / \delta\rfloor-1} \mathbb{E}\left\|\int_{0}^{\frac{\delta}{\epsilon}} S_{t-(\epsilon s+k \delta)}\left[f\left(X_{k \delta}^{\epsilon}, \hat{Y}_{s \epsilon+k \delta}^{\epsilon}\right)-\bar{f}\left(X_{k \delta}^{\epsilon}\right)\right] d s\right\|^{2} \\
= & \lfloor T / \delta\rfloor^{2} \epsilon^{2} \\
& \times \max _{0 \leq k \leq\lfloor T / \delta\rfloor-1} \int_{D} \mathbb{E}\left\{\int_{0}^{\frac{\delta}{\epsilon}} S_{t-(\epsilon s+k \delta)}\left[f\left(X_{k \delta}^{\epsilon}, \hat{Y}_{s \epsilon+k \delta}^{\epsilon}\right)-\bar{f}\left(X_{k \delta \delta}^{\epsilon}\right)\right] d s\right\}^{2} d \xi \\
= & 2\lfloor T / \delta\rfloor^{2} \epsilon^{2} \\
& \times \max _{0 \leq k \leq\lfloor T / \delta\rfloor-1} \int_{D} \mathbb{E}\left\{\int_{0}^{\frac{\delta}{\epsilon}} \int_{\tau}^{\frac{\delta}{\epsilon}} S_{t-(\epsilon s+k \delta)}\left[f\left(X_{k \delta}^{\epsilon}, \hat{Y}_{s \epsilon+k \delta}^{\epsilon}\right)-\bar{f}\left(X_{k \delta}^{\epsilon}\right)\right]\right. \\
& \left.\times S_{t-(\tau \epsilon+k \delta)}\left[f\left(X_{k \delta}^{\epsilon}, \hat{Y}_{\tau \epsilon+k \delta}^{\epsilon}\right)-\bar{f}\left(X_{k \delta}^{\epsilon}\right)\right] d s d \tau\right\} d \xi \\
:= & 2\lfloor T / \delta\rfloor^{2} \epsilon^{2} \max _{0 \leq k \leq\lfloor T / \delta\rfloor-1} \int_{0}^{\frac{\delta}{\epsilon}} \int_{\tau}^{\frac{\delta}{\epsilon}} \mathcal{J}_{k}(s, \tau) d s d \tau,
\end{aligned}
$$

with

$$
\begin{aligned}
& \mathcal{J}_{k}(s, \tau)=\mathbb{E} \int_{D} S_{t-(\epsilon s+k \delta)}\left[f\left(X_{k \delta}^{\epsilon}, \hat{Y}_{s \epsilon+k \delta}^{\epsilon}\right)-\bar{f}\left(X_{k \delta}^{\epsilon}\right)\right] \\
& \times S_{t-(\tau \epsilon+k \delta)}\left[f\left(X_{k \delta}^{\epsilon}, \hat{Y}_{\tau \epsilon+k \delta}^{\epsilon}\right)-\bar{f}\left(X_{k \delta}^{\epsilon}\right)\right] d \xi \\
& =\mathbb{E} \int_{D} S_{t-(\epsilon s+k \delta)}\left[f\left(X_{k \delta}^{\epsilon}, Y_{s}^{X_{k \delta}^{\varepsilon}, Y_{k \delta}^{\varepsilon}}\right)-\bar{f}\left(X_{k \delta}^{\epsilon}\right)\right] \\
& \times S_{t-(\tau \epsilon+k \delta)}\left[f\left(X_{k \delta}^{\epsilon}, Y_{\tau}^{X_{k \delta}^{\varepsilon}, Y_{k \delta}^{\varepsilon}}\right)-\bar{f}\left(X_{k \delta}^{\epsilon}\right)\right] d \xi \\
& =\mathbb{E} \int_{D}\left\{S_{t-(\tau \epsilon+k \delta)}\left[f\left(X_{k \delta}^{\epsilon}, Y_{\tau}^{X_{k \delta}^{\varepsilon}, Y_{k \delta}^{\varepsilon}}\right)-\bar{f}\left(X_{k \delta}^{\epsilon}\right)\right]\right. \\
& \left.\times \mathbb{E}^{Y_{\tau}^{X_{k \delta}^{\varepsilon}, Y_{k \delta}^{\varepsilon}}} S_{t-(s \epsilon+k \delta)}\left[f\left(X_{k \delta}^{\epsilon}, Y_{s-\tau}^{X_{k \delta}^{\varepsilon}, Y_{k \delta}^{\varepsilon}}\right)-\bar{f}\left(X_{k \delta}^{\epsilon}\right)\right]\right\} d \xi \\
& \leq\left\{\mathbb{E} \int_{D}\left(S_{t-(\tau \epsilon+k \delta)}\left[f\left(X_{k \delta}^{\epsilon}, Y_{\tau}^{X_{k \delta}^{\varepsilon}, Y_{k \delta}^{\varepsilon}}\right)-\bar{f}\left(X_{k \delta}^{\epsilon}\right)\right]\right)^{2} d \xi\right\}^{\frac{1}{2}} \\
& \times\left\{\mathbb{E} \int_{D}\left\{\mathbb{E}^{Y_{\tau}^{X_{k \delta}^{\varepsilon}, Y_{k \delta}^{\varepsilon}}}\left[S_{t-(s \epsilon+k \delta)}\left[f\left(X_{k \delta}^{\epsilon}, Y_{s-\tau}^{X_{k \delta}^{\varepsilon}, Y_{k \delta}^{\varepsilon}}\right)-\bar{f}\left(X_{k \delta}^{\epsilon}\right)\right]\right]\right\}^{2} d \xi\right\}^{\frac{1}{2}} \\
& \leq\left\{\mathbb{E}\left\|f\left(X_{k \delta}^{\epsilon}, Y_{\tau}^{X_{k \delta}^{\varepsilon}, Y_{k \delta}^{\varepsilon}}\right)-\bar{f}\left(X_{k \delta}^{\epsilon}\right)\right\|^{2}\right\}^{\frac{1}{2}} \\
& \times\left\{\mathbb{E}\left\|\mathbb{E}^{Y_{\tau}^{X_{k \delta}^{\varepsilon}, Y_{k \delta}^{\varepsilon}}}\left[f\left(X_{k \delta}^{\epsilon}, Y_{s-\tau}^{X_{k \delta}^{\epsilon}, Y_{k \delta}^{\varepsilon}}\right)-\bar{f}\left(X_{k \delta}^{\epsilon}\right)\right]\right\|^{2}\right\}^{\frac{1}{2}} .
\end{aligned}
$$

To proceed, by Appendix A.1 and the assumption that $f$ is bound, we get

$$
\mathcal{J}_{k}(s, \tau) \leq C\left\{\left(1+\mathbb{E}\left\|X_{k \delta}^{\varepsilon}\right\|^{2}+\mathbb{E}\left\|Y_{k \delta}^{\varepsilon}\right\|^{2}\right) e^{-\eta(s-\tau)}\right\}^{\frac{1}{2}} \leq C e^{-\frac{\eta}{2}(s-\tau)} .
$$


Therefore, by choosing $\delta=\delta(\varepsilon)$ such that $\frac{\delta}{\varepsilon}$ is sufficiently large, we have

$$
\begin{aligned}
\mathbb{E} \sup _{0 \leq t \leq T}\left\|\Xi_{11}(t)\right\|^{2} & \leq 2\lfloor T / \delta\rfloor^{2} \epsilon^{2} \max _{0 \leq k \leq\lfloor T / \delta\rfloor-1} \int_{0}^{\frac{\delta}{\epsilon}} \int_{\tau}^{\frac{\delta}{\epsilon}} \mathcal{J}_{k}(s, \tau) d s d \tau \\
& \leq 2\lfloor T / \delta\rfloor^{2} \epsilon^{2}\left(\frac{2}{\eta} \frac{\delta}{\varepsilon}-\frac{4}{\eta^{2}}+e^{\frac{-\eta}{2} \frac{\delta}{\varepsilon}}\right) \\
& \leq C \frac{\epsilon^{2}}{\delta^{2}}\left(\frac{2}{\eta} \frac{\delta}{\varepsilon}-\frac{4}{\eta^{2}}+e^{\frac{-\eta}{2} \frac{\delta}{\varepsilon}}\right) \\
& \leq C \frac{\epsilon}{\delta} .
\end{aligned}
$$

This completes the proof of Lemma 3.7.

\section{Acknowledgements}

The authors would like to thank the referees for their careful reading of the manuscript and for the clarifying comments, which lead to an improvement of the presentation of the paper.

\section{Competing interests}

The authors declare that they have no competing interests.

\section{Authors' contributions}

All authors contributed equally to the writing of this paper. All authors read and approved the final manuscript.

\section{Author details}

'School of Aeronautics, Northwestern Polytechnical University, Xi'an, 710072, China. ${ }^{2}$ Department of Applied Mathematics, Northwestern Polytechnical University, Xi'an, 710072, China.

\section{Publisher's Note}

Springer Nature remains neutral with regard to jurisdictional claims in published maps and institutional affiliations.

Received: 12 May 2017 Accepted: 26 August 2017 Published online: 08 September 2017

\section{References}

1. Larter, R, Steinmetz, C, Aguda, B: Fast-slow variable analysis of the transition to mixed-mode oscillations and chaos in the peroxidase reaction. J. Chem. Phys. 89, 6504-6514 (1988)

2. Krupa, $M$, et al.: Mixed-mode oscillations in a three time-scale model for the dopaminergic neuron. Chaos 18, Article ID 015106 (2008)

3. Dubbeldam, J, Krauskopf, B: Self-pulsations in lasers with saturable absorber: dynamics and bifurcations. Opt. Commun. 159, 325-338 (1999)

4. Khasminskii, R: On the averaging principle for stochastic differential Itô equations. Kybernetika 4, $260-279$ (1968)

5. Freidlin, M, Wentzell, A: Random Perturbations of Dynamical Systems. Springer, New York (1998)

6. Golec, J, Ladde, G: Averaging principle and systems of singularly perturbed stochastic differential equations. J. Math. Phys. 31, 1116-1123 (1990)

7. Xu, Y, Duan, J, et al.: An averaging principle for stochastic dynamical systems with Lévy noise. Physica D 240, 1395-1401 (2011)

8. Xu, Y, Pei, B, Li, Y: Approximation properties for solutions to non-Lipschitz stochastic differential equations with Lévy noise. Math. Methods Appl. Sci. 30, 2120-2131 (2015)

9. $\mathrm{Xu}, \mathrm{Y}, \mathrm{Pei}, \mathrm{B}, \mathrm{Wu}, \mathrm{J}$ : Stochastic averaging principle for differential equations with non-Lipschitz coefficients driven by fractional Brownian motion. Stoch. Dyn. 17(2), Article ID 1750013 (2017)

10. $X u, Y, G u o, R$, Liu, D, Zhang, H, Duan, J: Stochastic averaging principle for dynamical systems with fractional Brownian motion. Discrete Contin. Dyn. Syst., Ser. B 19(4), 1197-1212 (2014)

11. Xu, Y, Pei, B, Guo, R: Stochastic averaging for slow-fast dynamical systems with fractional Brownian motion. Discrete Contin. Dyn. Syst., Ser. B 20(7), 2257-2267 (2015)

12. $X u, Y, P e i, B, L i, Y$ : An averaging principle for stochastic differential delay equations with fractional Brownian motion. Abstr. Appl. Anal. 2014, Article ID 479195 (2014)

13. Pei, $B, X u, Y, Y i n, G$ : Averaging principles for SPDEs driven by fractional Brownian motions with random delays modulated by two-time-scale Markov switching processes. Stoch. Dyn. 18, Article ID 1850023 (2018)

14. Pei, $B, X u, Y, Y i n, G$ : Stochastic averaging for a class of two-time-scale systems of stochastic partial differential equations. Nonlinear Anal., Theory Methods Appl. 160, 159-176 (2017)

15. Golec, J: Stochastic averaging principle for systems with pathwise uniqueness. Stoch. Anal. Appl. 13, 307-322 (1995)

16. Wang, W, Roberts, A: Average and deviation for slow-fast stochastic partial differential equations. J. Differ. Equ. 253, $1265-1286$ (2012) 
17. Fu, H, Liu, J: Strong convergence in stochastic averaging for two-time-scales stochastic partial differential equations. J. Math. Anal. Appl. 384, 70-86 (2011)

18. Fu, H, Wang, L, Wang, Y, Liu, J: Strong convergence rate in averaging principle for stochastic FitzHugh-Nagumo system with two-time-scales. J. Math. Anal. Appl. 416, 609-628 (2014)

19. Applebaum, D: Lévy Processes and Stochastic Calculus, 2nd edn. Cambridge University Press, Cambridge (2009)

20. $\mathrm{Xu}, \mathrm{Y}$, Feng, J, et al.: Lévy noise induced switch in the gene transcriptional regulatory system. Chaos 23 , Article ID $013110(2013)$

21. $\mathrm{Xu}, \mathrm{Y}, \mathrm{Li}, \mathrm{J}$, et al.: Lévy noise-induced stochastic resonance in a bistable system. Eur. Phys. J. B 86, Article ID 198 (2013)

22. $L i, Y, X u, Y$, et al.: Lévy-noise-induced transport in a rough triple-well potential. Phys. Rev. E 94(4), Article ID 042222 (2016)

23. $X u, Y, L i, Y$, et al.: The switch in a genetic toggle system with Lévy noise. Sci. Rep. 6, Article ID 31505 (2016)

24. Wang, $Z, X u, Y$, Yang, H: Lévy noise induced stochastic resonance in an FHN model. Sci. China, Technol. Sci. 59(3), 371-375 (2016)

25. $\mathrm{Xu}, \mathrm{Y}, \mathrm{Li}, \mathrm{H}$, et al.: The estimates of the mean first exit time of a bistable system excited by Poisson white noise. J. Appl. Mech. 84(9), Article ID 091004 (2017)

26. Yin, C, Wen, Y, Zhao, Y: Optimal dividends problem with a terminal value for spectrally positive Lévy processes. Insur. Math. Econ. 53, 769-773 (2013)

27. Yin, C, Wen, Y, Zhao, Y: On the optimal dividend problem for a spectrally positive Lévy process. ASTIN Bull. 44, 635-651 (2014)

28. Wen, Y, Yin, C: Exit problems for jump processes having double-sided jumps with rational Laplace transforms. Abstr. Appl. Anal. 2014, Article ID 747262 (2014)

29. $L u, Y, W u, R$ : The differentiability of dividends function on jump-diffusion risk process with a barrier dividend strategy Front. Math. China 9, 1073-1088 (2014)

30. Dong, H, Liu, Z: The ruin problem in a renewal risk model with two-sided jumps. Math. Comput. Model. 57, 800-811 (2013)

31. Yin, C, Shen, Y, Wen, Y: Exit problems for jump processes with applications to dividend problems. J. Comput. Appl. Math. 245, 30-52 (2013)

32. $L u, Y, W u, R, X u, R$ : The joint distributions of some actuarial diagnostics for the jump-diffusion risk process. Acta Math. Sci. 30(3), 664-676 (2010)

33. Yin, $C$, Wen, $Y$, Zong, Z, Shen, Y: The first passage time problem for mixed-exponential jump processes with applications in insurance and finance. Abstr. Appl. Anal. 2014, Article ID 571724 (2014)

34. Xu, Y, Pei, B, Guo, G: Existence and stability of solutions to non-Lipschitz stochastic differential equations driven by Lévy noise. Appl. Math. Comput. 263, 398-409 (2015)

35. Pei, B, Xu, Y: Mild solutions of local non-Lipschitz neutral stochastic functional evolution equations driven by jumps modulated by Markovian switching. Stoch. Anal. Appl. 35, 391-408 (2017)

36. Pei, B, Xu, Y: Mild solutions of local non-Lipschitz stochastic evolution equations with jumps. Appl. Math. Lett. 52, 80-86 (2016)

37. Bouchaud, J, Georges, A: Anomalous diffusion in disordered media: statistic mechanics, models and physical applications. Phys. Rep. 195, 127-293 (1990)

38. Duan, J: An Introduction to Stochastic Dynamics. Cambridge University Press, Cambridge (2015)

39. Peszat, S, Zabczyk, J: Stochastic Partial Differential Equations with Lévy Noise. Encyclopedia of Mathematics and Its Applications. Cambridge University Press, Cambridge (2007)

40. DaPrato, G, Zabczyk, J: Stochastic Equations in Infinite Dimensions. Cambridge University Press, Cambridge (1992)

41. Duan, J, Wang, W: Effective Dynamics of Stochastic Partial Differential Equations. Elsevier, Amsterdam (2014)

42. Albeverio, S, Wu, J-L, Zhang, T: Parabolic SPDEs driven Poisson white noise. Stoch. Process. Appl. 74, 21-36 (1998)

43. Hausenblas, E: Existence, uniqueness and regularity of SPDEs driven by Poisson random measures. Electron. J. Probab. 10, 1496-1546 (2005)

44. Givon, D: Strong convergence rate for two-time-scale jump-diffusion stochastic differential systems. SIAM J. Multiscale Model. Simul. 6, 577-594 (2007)

45. $\mathrm{Xu}, \mathrm{J}, \mathrm{MiaO}, \mathrm{Y}$, et al.: Strong averaging principle for slow-fast SPDEs with Poisson random measures. Discrete Contin. Dyn. Syst., Ser. B 20, 2233-2256 (2015)

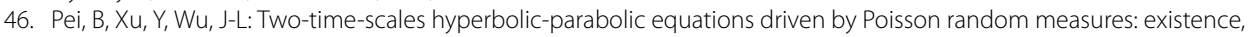
uniqueness and averaging principles. J. Math. Anal. Appl. 447, 243-268 (2017)

47. Chow, P: Stochastic Partial Differential Equations. Chapman \& Hall/CRC, Boca Raton (2014)

48. Pazy, A: Semigroups of Linear Operators and Applications to Partial Differential Equations. Springer, Berlin (2012)

49. Cerrai, S, Freidlin, M: Averaging principle for a class of stochastic reaction-diffusion equations. Probab. Theory Relat. Fields 144(1-2), 137-177 (2009)

50. Cerrai, S: A Khasminskii type averaging principle for stochastic reaction-diffusion equations. Ann. Appl. Probab. 19(3), 899-948 (2009) 